\title{
A Computationally Efficient Moment-Preserving Monte Carlo Electron Transport Method with Implementation in Geant4
}

\author{
D. A. Dixon ${ }^{1}$ \\ Los Alamos National Laboratory \\ P.O. Box 1663 MS P365 \\ Los Alamos, NM 87545 \\ A. K. Prinja ${ }^{2}$ \\ Department of Nuclear Engineering \\ MSC01 1120 \\ 1 University of New Mexico \\ Albuquerque, NM 87131-0001 \\ B. C. Franke ${ }^{3}$ \\ Sandia National Laboratories \\ Albuquerque, NM 87123
}

\begin{abstract}
This paper presents the theoretical development and numerical demonstration of a moment-preserving Monte Carlo electron transport method. Foremost, a full implementation of the Moment-Preserving (MP) method within the Geant4 particle simulation toolkit is demonstrated. Beyond implementation details, it is shown that the MP method is a viable alternative to the Condensed History $(\mathrm{CH})$ method for inclusion in current and future generation transport codes through demonstration of the key features of the method including: systematically controllable accuracy, computational efficiency, mathematical robustness, and versatility. A wide variety of results common to electron transport are presented illustrating the key features of the MP method. In particular, it is possible to achieve accuracy that is statistically indistinguishable from analog
\end{abstract}

\footnotetext{
${ }^{1}$ ddixon@lanl.gov

2 prinja@unm.edu

3 bcfrank@sandia.gov
}

Preprint submitted to Nuclear Instruments and Methods in Physics Research B July 14, 2015 
Monte Carlo, while remaining up to three orders of magnitude more efficient than analog Monte Carlo simulations. Finally, it is shown that the MP method can be generalized to any applicable analog scattering DCS model by extending previous work on the MP method beyond analytical DCSs to the partial-wave (PW) elastic tabulated DCS data.

Keywords: Electron transport, moment preservation, partial-wave differential cross sections, Monte Carlo simulation

\section{Introduction}

It is well known that analog or event-by-event Monte Carlo simulation [1] of electrons in complex material geometries is generally impractical because of highly peaked angular deflection and energy-loss differential cross sections

5 (DCS) and extremely small collision mean free paths (mfp) that result from Coulomb interactions between the incident electron and target nuclei and electrons. The high computational cost of analog electron transport has resulted in the development of a variety of approximate but more efficient approaches over the past 60 years, chief among these being the condensed history $(\mathrm{CH})$ Monte Carlo method [2]. In this approach, an electron is advanced in fixed spatial steps and its energy and angle are sampled at the end of a step from decoupled and precomputed angular deflection and energy-loss distributions which, however, are based on approximate infinite medium multiple scattering and multiple excitation/ionization theories. It is necessary to impose pathlength and transverse displacement corrections at the end of the step and algorithmic modifications at material interfaces and free-surface boundaries, where the infinite medium distributions become invalid, in order to achieve acceptable accuracy in the condensed history method. $[3,4,5]$.

Here we present an alternative technique that uses reduced order physics 20 (ROP) approximations to the Boltzmann collision operator for elastic and inelastic interactions to restore the correct transport mechanics while enabling efficient single-event Monte Carlo simulation of electron transport. Earlier work 
along these lines used decomposition of the underlying differential cross sections [6] to isolate and specially treat the forward peaked components, using approximations such as Fokker-Planck (FP) [7], Boltzmann Fokker-Planck (BFP) [8, 9], Generalized Fokker-Planck (GFP) [10, 11], and Generalized Boltzmann FokkerPlanck (GBFP) $[12,13]$. As will become apparent in the ensuing sections, these and other related methods fall under the general scheme of moment-preserving (MP) methods where the analog or true differential cross section is replaced by one that attempts to preserve only a sufficient number of important properties of the interaction physics, as measured by angular and energy-loss moments. Various moment-preserving strategies, where a sufficient number of low-order moments are exactly preserved while all higher-order moments are approximated in terms of these lower-order moments, are employed in this approach 35 to construct ROP DCSs that are less singular, with mfps longer than those characteristic of the analog DCS. A key feature of our formulation is that by systematically increasing the number of moments preserved, any desired accuracy can be achieved for integrated quantities, such as dose [12], reflection and transmission yields, as well as distributed quantities such as energy and angular distributions $[14,15]$.

Although aspects of our approach and preliminary results have been reported before [12], here we describe the method in generality, extend the earlier work to accommodate the tabulated PW elastic scattering DCSs [16] that are considered the most accurate representation of elastic scattering currently available, and, 45 perhaps most significantly, demonstrate implementation of the MP-method in the production code Geant4 [17].

The scope of the paper is as follows. In the next section, we discuss the electron elastic and inelastic scattering physics and introduce the analog DCSs that are used to generate the analog benchmarks and to construct the ROP DCSs central to the method. The analog transport model is described in the following section and is followed by a thorough discussion of the MP method. Algorithmic details are then presented, and this is followed by a description of the implementation of the method in Geant4. Finally, extensive numerical 
results are presented for a variety of problems, and the paper concludes with some remarks on the current status of the method and future directions.

\section{Electron Elastic and Inelastic Scattering Physics}

In this paper, we are concerned with the interactions that render analog Monte Carlo electron transport computationally inefficient. That is, elastic collisions with atomic nuclei (primary source of deflection) and inelastic collisions with atomic electrons (primary source of energy-loss). These interactions are characterized by highly peaked DCSs about small changes in direction and small energy transfers or energy losses resulting in extremely large total cross sections or short mfps on the order of microns. In the following sections, we introduce the elastic and inelastic analog physics models used in this study.

\subsection{Partial-wave elastic differential cross section}

The PW DCSs are numerically evaluated using the ELSEPA code [16] and are considered accurate representations of the elastic scattering of electrons by atomic nuclei at energies above roughly 1-keV. The PW DCSs utilized in this work are similar to those included in the ICRU-ELSEP database [18] with an exception being that the PW DCSs were evaluated on an energy grid with 16 logarithmically spaced points between $10-\mathrm{MeV}$ and $20-\mathrm{MeV}$ and 107 logarithmically spaced points between $1-\mathrm{keV}$ and $10-\mathrm{MeV}$. A log-log linear interpolation scheme [5] was used to obtain both the total cross section and the DCS for a given energy between grid points.

The elastic collisions with nuclei considered herein deflect the incident electron through some scattering angle, $\theta$. While some energy is transferred to the nucleus during an elastic collision, the mass of the nucleus is so large in comparison to the electron mass that the energy transferred to the nucleus is assumed negligible. As mentioned, elastic scattering DCSs are extremely peaked about very small deflection angles and in some cases vary up to 28 orders of magnitude resulting in extremely short mfps as small as fractions of a micron. This 
behavior is seen in Figs. 1a and 1b where PW DCSs are presented for aluminum and gold at various energies.

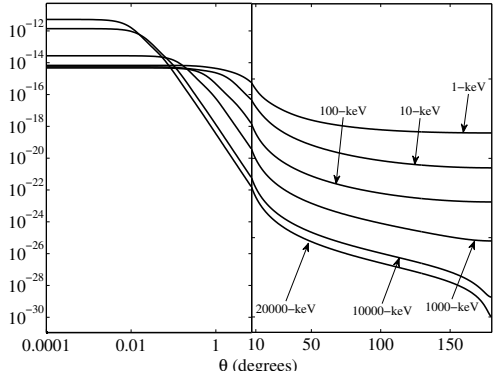

(a) Aluminum

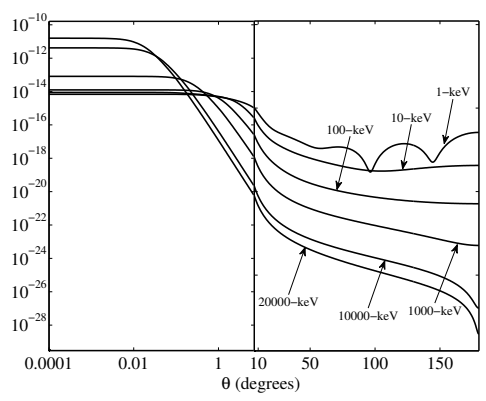

(b) Gold

Figure 1: Partial-wave DCSs for elastic scattering of electrons with energies between 1-keV and 20000-keV by (a) aluminum and (b) gold nuclei.

\subsection{Möller inelastic differential cross section}

The Möller inelastic DCS [19] accounts for energy transferred to atomic electrons resulting in ionization of the target atom. In the Möller DCS, binding energies for the electron shells are neglected; therefore, Möller is an approximation over all of the shells where the minimum energy transfer $Q_{\min }$ is assumed to be the mean ionization potential $I_{\mathrm{MeV}}[20]$. The resulting inelastic DCS in which the incident electron with rest mass of $m_{0} c^{2}$ and kinetic energy $E$ transfers energy $Q$ to the slower electron is given by

$\Sigma_{i n}(E, Q)= \begin{cases}\frac{2 \pi r_{e}^{2} Z m_{0} c^{2}}{\beta^{2}}\left[\frac{1}{Q^{2}}+\frac{1}{(E-Q)^{2}}+\frac{1}{\left(E+m_{0} c^{2}\right)}-\frac{m_{0} c^{2}\left(2 E+m_{0} c^{2}\right)}{Q(E-Q)\left(E+m_{0} c^{2}\right)^{2}}\right] & , Q \in\left[Q_{\text {min }}, Q_{\text {max }}\right] \\ 0 & , \text { otherwise, }\end{cases}$

where the upper bound is $Q_{\max }=E / 2$. Like the elastic scattering DCS, the inelastic DCS is peaked about small energy transfers or $Q_{m i n}$. The Möller DCS is not considered an accurate representation of inelastic collisions except for large energy transfers. In fact, the first moment of the Möller DCS, that is the stopping power, fails to reproduce the stopping powers given by the ICRU Report 
37 [18]. Here, we renormalize the Möller DCS such that the first moment is in agreement with the stopping powers in ICRU Report 37, or $S(E)$. Specifically, the renormalized Möller DCS is given by

$$
\hat{\Sigma}_{i n}(E, Q)=\kappa(E) \Sigma_{i n}(E, Q),
$$

where, $\kappa$ is the renormalization coefficient or

$$
\kappa(E)=\frac{S(E)}{Q_{1}(E)} .
$$

In Eq. (3), $Q_{1}$ is the definition of the stopping power [19], or the first energy-loss moment of the inelastic DCS and is given by

$$
Q_{1}=\int_{Q_{\min }}^{Q_{\max }} Q \Sigma_{i n}(E, Q) d Q .
$$

Given a description of the electron interaction physics under consideration, we turn our attention to the corresponding transport equation.

\section{Analog Transport Model}

The linear Boltzmann equation for the angular flux of electrons $\psi(\vec{r}, E, \vec{\Omega})$ with position $\vec{r}(x, y, z)$, energy $E$, and direction $\vec{\Omega}(\phi, \mu)$ is expressed by:

$$
\Omega \cdot \nabla \psi(\vec{r}, E, \vec{\Omega})=H^{B} \psi(\vec{r}, E, \vec{\Omega}),
$$

where the Boltzmann collision operator is defined as:

$$
\begin{aligned}
H^{B} \psi(\vec{r}, E, \vec{\Omega}) & =\int_{0}^{\infty} d E^{\prime} \int_{4 \pi} d \Omega^{\prime}\left[\Sigma_{e l}\left(\vec{r}, E^{\prime} \rightarrow E, \vec{\Omega}^{\prime} \cdot \vec{\Omega}\right)\right. \\
& \left.+\Sigma_{i n}\left(\vec{r}, E^{\prime} \rightarrow E, \vec{\Omega}^{\prime} \cdot \vec{\Omega}\right)\right] \psi\left(\vec{r}, E^{\prime}, \vec{\Omega}^{\prime}\right)-\Sigma_{t}(\vec{r}, E) \psi(\vec{r}, E, \vec{\Omega}),
\end{aligned}
$$

where $\Sigma_{t}(\vec{r}, E)=\Sigma_{e l, 0}+\Sigma_{i n, 0}$. Eq. (5) is subject to the following boundary condition:

$$
\psi(\vec{r}, E, \phi, \mu)=\psi^{b}(\vec{r}, E, \phi, \mu), \vec{r} \in \partial V, \vec{\Omega}(\phi, \mu) \cdot \vec{n}<0,0<E<\infty
$$

For electrons, elastic and inelastic scattering are treated separately as indicated in Eq. (6). Furthermore, it is assumed that elastic scattering occurs 
without energy loss and angular deflection from inelastic scattering is given by kinematics, so the collision operator can be expressed as

$$
H^{B} \psi(\vec{r}, E, \vec{\Omega})=H_{e l}^{B} \psi(\vec{r}, E, \vec{\Omega})+H_{i n}^{B} \psi(\vec{r}, E, \vec{\Omega})
$$

where

$$
H_{e l}^{B} \psi(\vec{r}, E, \vec{\Omega})=\int_{4 \pi} d \Omega^{\prime} \Sigma_{e l}\left(\vec{r}, E, \vec{\Omega}^{\prime} \cdot \vec{\Omega}\right) \psi\left(\vec{r}, E, \vec{\Omega}^{\prime}\right)-\Sigma_{e l, 0}(\vec{r}, E) \psi(\vec{r}, E, \vec{\Omega})
$$

and

$$
\begin{aligned}
H_{i n}^{B} \psi(\vec{r}, E, \vec{\Omega})= & \int_{4 \pi} d \Omega^{\prime} \int_{0}^{\infty} d E^{\prime} \Sigma_{i n}\left(\vec{r}, E^{\prime} \rightarrow E\right) \delta\left[\vec{\Omega}^{\prime}-f\left(E^{\prime}, Q\right)\right] \psi\left(\vec{r}, E^{\prime}, \vec{\Omega}^{\prime}\right) \\
& -\Sigma_{i n, 0}(\vec{r}, E) \psi(\vec{r}, E, \vec{\Omega}),
\end{aligned}
$$

where the angle of the primary electron is

$$
f(E, Q)=\sqrt{\frac{E-Q}{E} \frac{E+2 m_{e} c^{2}}{E-Q+2 m_{e} c^{2}}}
$$

and the angle of the secondary electron is

$$
g(E, Q)=\sqrt{\frac{Q}{E} \frac{E+2 m_{e} c^{2}}{Q+2 m_{e} c^{2}}} .
$$

In the event that secondary electron production is neglected, deflection of the primary electron from inelastic scattering is also neglected and Eq. (10) reduces to

$$
H_{e l}^{B} \psi(\vec{r}, E, \vec{\Omega})=\int_{0}^{\infty} d E^{\prime} \Sigma_{i n}\left(\vec{r}, E^{\prime} \rightarrow E\right) \psi\left(\vec{r}, E^{\prime}, \vec{\Omega}\right)-\Sigma_{i n, 0}(\vec{r}, E) \psi(\vec{r}, E, \vec{\Omega}) .
$$

As previously mentioned, direct numerical solution of Eq. (5) is not realistic for most applications of interest because of the large total cross sections, $\Sigma_{e l, 0}$ and $\Sigma_{i n, 0}$. Because the computational effort is related to the number of collisions simulated per particle history, simulation of Coulomb collisions are far more computationally intensive than the interactions characterizing neutral particles. 


\section{Moment-Preserving Method}

In the MP method, a reduced order physics (ROP) transport equation is formed by replacing the analog DCSs in Eqs. (9) and (13) with ROP DCSs, $\tilde{\Sigma}$. That is,

$$
\tilde{H}_{e l}^{B} \psi(\vec{r}, E, \vec{\Omega})=\int_{4 \pi} d \Omega^{\prime} \tilde{\Sigma}_{e l}\left(\vec{r}, E, \vec{\Omega}^{\prime} \cdot \vec{\Omega}\right) \psi\left(\vec{r}, E, \vec{\Omega}^{\prime}\right)-\tilde{\Sigma}_{e l, 0}(\vec{r}, E) \psi(\vec{r}, E, \vec{\Omega})
$$

and

$$
\tilde{H}_{i n}^{B} \psi(\vec{r}, E, \vec{\Omega})=\int_{0}^{\infty} d E^{\prime} \tilde{\Sigma}_{i n}\left(\vec{r}, E^{\prime} \rightarrow E, \vec{\Omega}\right) \psi\left(\vec{r}, E^{\prime}, \vec{\Omega}\right)-\tilde{\Sigma}_{i n, 0}(\vec{r}, E) \psi(\vec{r}, E, \vec{\Omega}) .
$$

Although simply replacing the analog DCS with an ROP DCS may seem triv-

ial or even arbitrary, there is no absence of rigor in this method. Particularly, much consideration is given to the form and properties of the ROP DCSs. The ROP DCSs are constructed such that they are smoother or less-peaked functions of deflection angle and energy loss and have significantly longer mfps than the analog DCSs. Thus, the ROP collision operators in Eqs. (14) and (15) have better properties than the analog collision operators, especially, from an efficiency standpoint.

Beyond efficiency, there are additional properties of the ROP collision operators that set this method apart from other approximate methods. For example, one of the unique characteristics of this method is that the integral form of the Boltzmann collision operators are maintained. Therefore, the description of the underlying transport mechanics is not lost, specifically, the correct Markovian feature of exponentially distributed collision sites [12]. Therefore, special algorithms for handling material and vacuum interfaces are not required.

Moreover, exact treatment of collisions as Markov processes and less-peaked DCSs with longer mfps make it practical to simulate transport with a singleevent method. Implementation of single-event methods are very straightforward compared to other methods like $\mathrm{CH}$ that are considerably more complicated. In fact, Monte Carlo codes with pre-existing single-event algorithms do not require 
any retrofitting when implementing the MP method, because this method treats electrons like neutral particles.

The MP method is efficient and implementation is straightforward, but there has been no mention of accuracy. This method must not only be competitive with, and potentially superior to $\mathrm{CH}$ with regard to efficiency and simplicity, but accuracy as well, and in many cases it is. Unlike $\mathrm{CH}$, which introduces inherent and irreducible limits on accuracy as a result of the underlying theory, accuracy is systematically controllable. This is largely a result of the momentpreserving strategies that are central to this approach. The moment-preserving strategy is motivated by Lewis theory [21, 22], where Lewis showed that one can relate space-angle moments of the angular flux to momentum-transfer moments of the elastic scattering DCS. In addition, the eigenvalues of the elastic collision operator are directly dependent on the momentum-transfer moments. For these reasons, it is prudent to construct an ROP DCS that preserves moments of the analog DCS.

Given the relationship between the ROP DCS and the analog DCS moments, the following moment-preservation constraints are a natural choice when constructing an ROP DCS. If the analog elastic scattering moments are given by

$$
\Sigma_{e l, \ell}=2 \pi \int_{-1}^{1} P_{\ell}\left(\mu_{0}\right) \Sigma_{e l}\left(\mu_{0}\right) d \mu_{0},
$$

the moment preserving constraint is

$$
\Sigma_{e l, \ell}=\tilde{\Sigma}_{e l, \ell}, \quad \ell=1,2, \ldots L
$$

and the higher order moments are functions of the lower order moments

$$
\Sigma_{e l, \ell}=f\left(\Sigma_{e l, 1}, \Sigma_{e l, 2}, \ldots, \Sigma_{e l, L}\right), \quad \ell>L .
$$

For inelastic scattering the moments are given by

$$
\Sigma_{i n, j}=\int_{Q_{\min }}^{Q_{\max }} Q^{j} \Sigma_{i n}(Q) d Q .
$$

The moment preserving constraint is similar and given by

$$
\Sigma_{i n, j}=\tilde{\Sigma}_{i n, j}, \quad j=1,2, \ldots J .
$$


Again, the higher order moments are functions of the lower order moments

$$
\Sigma_{i n, j}=f\left(\Sigma_{i n, 1}, \Sigma_{i n, 2}, \ldots, \Sigma_{i n, J}\right), \quad j>J .
$$

By constructing an ROP DCS that preserves moments of the analog DCS, one

can systematically control the accuracy of the ROP DCS models. That is, improvements in accuracy are achieved by simply preserving more moments of the analog DCS. In addition, the higher order moments are functions of the exact lower order moments, so the higher order moments are good approximations rather than being neglected as is the case for other ROP models like FokkerPlanck.

The following sections present the two ROP DCS models that are used in this paper. Given the discrete ROP DCS model, we present a corresponding derivation of the ROP transport model for a discrete elastic and inelastic ROP DCS to emphasize the difference in the ROP transport model and the analog model. Lastly, the details of the ROP DCS construction process for each model are presented.

\subsection{Reduce order physics differential cross-section models}

In this section, we present two forms of the elastic and inelastic ROP DCSs that are demonstrated herein: the discrete DCS and the hybrid DCS. The discrete DCS is a superposition of discrete points and weights. One of the benefits of the discrete DCS is the simple form of the DCS. The discrete DCS is simple to sample and requires significantly less memory requirements than DCS data because only a few points and weights are required for most problems of interest. The accuracy and efficiency of the discrete DCS are especially promising when calculating integral quantities like dose [12]. We define the discrete DCS for elastic scattering as

$$
\tilde{\Sigma}_{e l}\left(\vec{r}, E, \mu_{0}\right)=\sum_{n=1}^{N} \frac{\alpha_{n}(E)}{2 \pi} \delta\left[\mu_{0}-\zeta_{n}\right],
$$

and for inelastic scattering as

$$
\tilde{\Sigma}_{i n}(\vec{r}, E, Q)=\sum_{n=1}^{N} \beta_{n}(E) \delta\left[Q-\gamma_{n}\right] .
$$


The one drawback of the discrete DCS is the presence of discrete artifacts [23, 15], especially, if the discrete DCS is used when calculating differential quantities in thin slabs. However, discrete artifacts can be mitigated by use of the hybrid DCS, while still achieving efficiency gains.

The hybrid DCS is a superposition of both discrete points and weights and a smooth function represented by an analog DCS. In previous work [14], the smooth component was represented by the SR DCS over $[-1,1]$. The screening parameter was artificially selected such that the smooth component was less peaked near one. Moments of the smooth component are then subtracted from the analog DCS moments and this difference is then used to generate the discrete scattering angles. In this work, a slightly different representation was chosen where the tail is represented exactly by the analog model up to some cut-off point, $\mu_{0}^{*}$. Beyond the cut-off point or for $\mu_{0} \in\left[\mu_{0}^{*}, 1\right]$ a discrete representation is used. The resulting hybrid DCS is:

$$
\tilde{\Sigma}_{e l}\left(\vec{r}, E, \mu_{0}\right)=\Sigma_{e l}^{S}\left(E, \mu_{0}\right)+\sum_{n=1}^{N} \frac{\alpha_{n}(E)}{2 \pi} \delta\left[\mu_{0}-\zeta_{n}\right],
$$

where $\Sigma_{e l}^{S}\left(E, \mu_{0}\right)$ is an analog DCS for $\mu_{0} \in\left[-1, \mu_{0}^{*}\right)$ and otherwise zero. The cut-off, $\mu_{0}^{*}$, is typically chosen to be near the peak, that is unity, to gain the benefit of the properties of the discrete DCS, while capturing the large-angle scattering exactly by the analog DCS. For inelastic scattering, the cut-off, $Q^{*}$, is selected near the peak, that is $Q_{\min }$, for the aforementioned reasons.

From an implementation standpoint, there is little difference between the discrete and hybrid DCS. It requires the ability to sample hard collisions from the analog DCS and soft collisions from the discrete DCS. The only difference in generating the discrete DCS versus the hybrid DCS is that the moments are now defined over a partial interval corresponding to the peak.

Given the form of the ROP DCSs, a derivation of the ROP collision operators that comprise the ROP transport equation is presented. 


\subsection{Derivation of the reduced order physics collision operators}

The ROP DCS is constructed such that the singular contribution to inscatter and outscatter cancel (similar to the FP operator [24]). Ultimately, the purpose of constructing such a DCS is that the resulting ROP transport equation can be solved accurately and efficiently using single-scatter models. To be clear, a derivation of the elastic and inelastic ROP collision operators is presented. A derivation of both operators is presented because there is a subtle difference between the two that deserves some attention. For the sake of simplicity, the discrete DCS is used, but the same ideas carry over to any ROP DCS.

The starting point is the elastic collision operator. Substitution of Eq. (22) into Eq. (9) gives

$$
\tilde{H}_{e l}^{B}=\int_{4 \pi} d \Omega^{\prime} \sum_{n=1}^{N+1} \frac{\alpha_{n}(E)}{2 \pi} \delta\left[\mu_{0}-\zeta_{n}\right] \psi\left(\vec{r}, E, \vec{\Omega}^{\prime}\right)-\left(\sum_{n=1}^{N+1} \alpha_{n}(E)\right) \psi(\vec{r}, E, \vec{\Omega}) .
$$

It is required that the discrete point $\zeta_{N+1}=1$. Now, this point is intentionally separated from the remaining $N$ points and weights in the inscatter and outscatter terms. That is,

$$
\begin{aligned}
\tilde{H}_{e l}^{B}= & \int_{4 \pi} d \Omega^{\prime} \sum_{n=1}^{N} \frac{\alpha_{n}(E)}{2 \pi} \delta\left[\mu_{0}-\zeta_{n}\right] \psi\left(\vec{r}, E, \vec{\Omega}^{\prime}\right)-\left(\sum_{n=1}^{N} \alpha_{n}(E)\right) \psi(\vec{r}, E, \vec{\Omega}) \\
& +\int_{4 \pi} d \Omega^{\prime} \frac{\alpha_{N+1}(E)}{2 \pi} \delta\left[\mu_{0}-\zeta_{N+1}\right] \psi\left(\vec{r}, E, \vec{\Omega}^{\prime}\right)-\alpha_{N+1} \psi(\vec{r}, E, \vec{\Omega}) .
\end{aligned}
$$

If it can be shown that the last two terms in Eq. (26) indeed cancel, the resulting ROP transport equation will have an elastic scattering kernel that is significantly less peaked with reduced total cross section because $\alpha_{N+1}$ no longer contributes to the total cross section. It is not difficult to show that the last two terms cancel because $\mu_{0}=\overrightarrow{\Omega^{\prime}} \cdot \vec{\Omega}=1$ if and only if $\vec{\Omega}^{\prime}=\vec{\Omega}$ (see ref. [25] for details). Therefore, the following is true

$$
\begin{aligned}
\int_{4 \pi} d \overrightarrow{\Omega^{\prime}} \frac{\alpha_{n}(E)}{2 \pi} \delta\left[\mu_{0}-1\right] \psi\left(\vec{r}, E, \vec{\Omega}^{\prime}\right) & =\int_{4 \pi} d \mu^{\prime} \frac{\alpha_{N+1}}{2 \pi}(E) \delta\left[\overrightarrow{\Omega^{\prime}} \cdot \vec{\Omega}-1\right] \psi\left(\vec{r}, E, \overrightarrow{\Omega^{\prime}}\right) \\
& =\alpha_{N+1}(E) \psi(\vec{r}, E, \vec{\Omega})
\end{aligned}
$$

This term clearly cancels with the last term in Eq. (26). The inelastic collision operator is now shown. 
The derivation of the inelastic ROP collision operator is easier to follow after a change of variables from $Q$ to $\left(E^{\prime}-E\right)$. This gives

$$
\tilde{\Sigma}_{i n}\left(\vec{r}, E^{\prime} \rightarrow E\right)=\sum_{n=1}^{N} \beta_{n}(E) \delta\left[\left(E^{\prime}-E\right)-\gamma_{n}\right] .
$$

Again, Eq. (28) is substituted into Eq. (10) and the argument of the delta function is rewritten as $E^{\prime}-\left(E+\gamma_{n}\right)$ which gives

$\tilde{H}_{i n}^{B}=\int_{0}^{\infty} d E^{\prime} \sum_{n=1}^{N+1} \beta_{n}(E) \delta\left[E^{\prime}-\left(E+\gamma_{n}\right)\right] \psi(\vec{r}, E, \vec{\Omega})-\left(\sum_{n=1}^{N+1} \beta_{n}(E)\right) \psi(\vec{r}, E, \vec{\Omega})$.

Now, the singular component or the $N+1$ term is separated from the inscatter and the outscatter, the inelastic ROP collision operator becomes

$$
\begin{aligned}
\tilde{H}_{e l}^{B}= & \int_{0}^{\infty} d E^{\prime} \sum_{n=1}^{N} \beta_{n}(E) \delta\left[E^{\prime}-\left(E+\gamma_{n}\right)\right] \psi(\vec{r}, E, \vec{\Omega})-\left(\sum_{n=1}^{N} \beta_{n}(E)\right) \psi(\vec{r}, E, \vec{\Omega}) \\
& +\int_{0}^{\infty} d E^{\prime} \beta_{N+1}(E) \delta\left[E^{\prime}-\left(E+\gamma_{N+1}\right)\right] \psi(\vec{r}, E, \vec{\Omega})-\beta_{N+1}(E) \psi(\vec{r}, E, \vec{\Omega}) .
\end{aligned}
$$

Carrying out the integration over the $N+1$ term results in the following singular contributions to inscatter and outscatter

$$
\gamma_{N+1}(E) \psi\left(\vec{r}, E+\chi_{N+1}, \vec{\Omega}\right)-\gamma_{N+1}(E) \psi(\vec{r}, E, \vec{\Omega})=0
$$

because $\chi_{N+1}=0$.

\section{Generation of the discrete and hybrid differential cross sections}

The procedure for constructing both the discrete and hybrid DCS from ana$\log$ DCS moments is described. The form of the moment preservation constraints in Eqs. (17) and (20) is unstable to direct numerical inversion, so another approach similar to generation of Radau quadrature is taken [26]. Initially, atten-

tion is given to the discrete elastic DCS and then the discussion is extended to the discrete inelastic DCS and the hybrid DCS.

It is of interest to obtain a DCS that satisfies the moment constraint in Eq. (17). Given the discrete elastic DCS in Eq. (22), a system of equations 
Eq. (22) into the right-hand-side of Eq. (17) results in the following system of equations:

$$
\begin{aligned}
\Sigma_{e l, \ell} & =\tilde{\Sigma}_{e l, \ell} \\
& =2 \pi \int_{-1}^{1} d \mu_{0} P_{\ell}\left(\mu_{0}\right) \tilde{\Sigma}_{e l}\left(E, \mu_{0}\right) \\
& =\sum_{n=1}^{N} \alpha_{n}(E) \int_{-1}^{1} d \mu_{0} P_{\ell}\left(\mu_{0}\right) \delta\left[\mu_{0}-\zeta_{n}\right] \\
& =\sum_{n=1}^{N} \alpha_{n}(E) P_{\ell}\left(\zeta_{n}\right) .
\end{aligned}
$$

A total of $L=2 N$ equations are necessary because there are $2 N$ unknowns,. That is,

$$
\begin{aligned}
\Sigma_{e l, 1} & =\alpha_{1}(E) P_{1}\left(\zeta_{1}\right)+\alpha_{2}(E) P_{1}\left(\zeta_{2}\right)+\ldots+\alpha_{2 N}(E) P_{1}\left(\zeta_{2 N}\right) \\
\Sigma_{e l, 2} & =\alpha_{1}(E) P_{2}\left(\zeta_{1}\right)+\alpha_{2}(E) P_{2}\left(\zeta_{2}\right)+\ldots+\alpha_{2 N}(E) P_{2}\left(\zeta_{2 N}\right) \\
& \vdots \\
\Sigma_{e l, 2 N} & =\alpha_{1}(E) P_{2 N}\left(\zeta_{1}\right)+\alpha_{2}(E) P_{2 N}\left(\zeta_{2}\right)+\ldots+\alpha_{2 N}(E) P_{2 N}\left(\zeta_{2 N}\right)
\end{aligned}
$$

The system formed in Eq. (33) emphasizes the requirement that $\zeta_{n}$ and $\alpha_{n}$ are obtained such that Legendre moments of the analog DCS are preserved. The system is then recast into one encountered when generating Gauss-Radau Quadrature for a non-classical weight function [27, 28]. That is,

$$
\Sigma_{e l, \ell}=\sum_{n=1}^{N} \alpha_{n}(E) P_{\ell}\left(\zeta_{n}\right)+\alpha_{N+1}(E) P_{\ell}\left(\zeta_{N+1}=1\right)
$$

which is a Gauss-Radau Quadrature system for a non-classical weight function, where in this case, the weight function is the analog DCS. Note that an additional unknown, $\alpha_{N+1}$, is added in Eq. (34) and multiplied by $P_{\ell}\left(\zeta_{N+1}=1\right)$. This is indicative of Radau quadrature and an expression for determining $\alpha_{N+1}$ is given below. A Radau approach is selected rather than standard Gauss quadrature because Radau ensures that one point will correspond to the peaked component of the DCS (that is, $\zeta_{N+1}=1$ ). Once the discrete points and weights 
are obtained, the peaked component is eliminated, thus, reducing the total cross section after renormalizing the discrete DCS. This is equivalent to satisfying the moment preservation constraints given in Eqs. (17) and (20).

To obtain the points and weights, coefficients of monic Legendre polynomials $\left(\alpha_{j}\right.$ and $\left.\beta_{j}\right)$ are mapped to the coefficients of polynomials orthogonal to the analog DCS $\left(a_{j}\right.$ and $\left.b_{j}\right)$. The algorithm for this mapping is referred to as the modified Chebyshev algorithm (MCA) [28] and requires $2 N-1$ moments of the analog DCS and $2 N-2$ coefficients of monic Legendre polynomials. Given a successful mapping and the resulting coefficients, $a_{j}$ and $b_{j}$, the Golub and Welsch algorithm [29] is used to obtain the eigenvalues of the Jacobi matrix. The Jacobi matrix is a tridiagonal matrix where the diagonal is set to $a_{i}$ and the off-diagonals are set to $\sqrt{b_{i}}$. The eigenvalues of the Jacobi matrix are the points and the first entry of each corresponding eigenvector squared are the weights. That is, $\zeta_{n}=\lambda_{n}(J)$ and $\alpha_{n}=\left(V_{n, 1}\right)^{2}$, where $\mathrm{V}$ is a eigenvector matrix. The application of the Golub and Welsch algorithm to the aforementioned Jacobi matrix will result in Gauss Quadrature and must be modified according to Golub [27] for Radau quadrature. Therefore, the Jacobi matrix is modified such that

$$
J_{N+1}=\left[\begin{array}{cc}
J_{N} & b_{N} \vec{e}_{N} \\
b_{N} \vec{e}_{N}^{T} & a_{N+1}
\end{array}\right],
$$

where

$$
a_{N+1}=1-b_{N} \frac{p_{N-1}(1)}{p_{N}(1)} .
$$

Application of the Golub and Welsch algorithm to Eq. (35) will result in $N+1$ points and $N+1$ weights normalized to unity. To obtain the final discrete DCS, the $N+1$ point and weight is eliminated and the remaining weights are then scaled by the analog total cross section or $\Sigma_{e l, 0}$. The total cross section for the discrete DCS is then

$$
\tilde{\Sigma}_{e l, 0}(\vec{r}, E)=\sum_{n=1}^{N} \alpha_{n}(E),
$$

which does not include the $N+1$ weight. The total cross section in Eq. (37) is significantly reduced depending on the order of the discrete DCS, the particle 
energy, and the target material, thus, extending the mfp. This completes the process of generating a discrete elastic DCS.

The process of generating a discrete inelastic DCS is similar. To use the same quadrature tools, the inelastic DCS must be mapped to an elastic DCS because the bounds on the elastic DCS are ideal for these tools (that is, $[-1,1]$ ). Given a mapping, the moments of the inelastic DCS are related to Legendre moments of an ROP elastic DCS. Points on $(-1,1]$ are generated with corresponding weights and then mapped back to $\left[0, Q_{\max }\right)$. The mapping is

$$
Q(\mu)=\frac{Q_{\max }}{2}(1-\mu)
$$

and the resulting relationship between the moments is

$$
\hat{\Sigma}_{e l, \ell}=\sum_{j=0}^{\ell} c_{j}^{\ell} \frac{(-1)^{j}}{j !}\left(\frac{2}{Q_{\max }}\right)^{j} \Sigma_{i n, j},
$$

where

$$
c_{j}^{\ell}=\frac{1}{2^{j} j !} \prod_{i=0}^{j-1}[l(l+1)-i(i+1)] .
$$

This summarizes the process of generating the discrete elastic and inelastic DCS. the hybrid DCS.

To generate the discrete points and weights for the hybrid DCS a cut-off value is selected. It should be selected such that additional accuracy is gained while still maintaining efficient runtimes. Although, this is problem dependent and mostly a heuristic exercise.

Given a cut-off, the following moments are used to generate the discrete points and weights:

$$
\Sigma_{e l, \ell}^{D}=2 \pi \int_{\mu_{0}^{*}}^{1} P_{\ell}\left(\mu_{0}\right) \Sigma_{e l}\left(\mu_{0}\right) d \mu_{0}
$$

and

$$
\Sigma_{i n, j}^{D}=\int_{Q_{m i n}}^{Q^{*}} Q^{j} \Sigma_{i n}(Q) d Q .
$$

In both cases, to use the DCS generation tools, the moments must be mapped to the appropriate domain $[-1,1]$ just as for the discrete inelastic DCS. For the 
inelastic hybrid DCS the mapping does not change significantly from Eqs. (38) and (39) and is

$$
Q(\mu)=\frac{1}{2} Q^{*}(1-\mu),
$$

where $\mu$ is on $[-1,1]$ and $Q$ is on $\left[0, Q^{*}\right]$. Given this mapping, the moments are related by

$$
\hat{\Sigma}_{e l, \ell}=\sum_{j=0}^{\ell} c_{j}^{\ell} \frac{(-1)^{j}}{j !}\left(\frac{2}{Q^{*}-Q_{\min }}\right)^{j} \sum_{k=0}^{j}\left(\begin{array}{l}
j \\
k
\end{array}\right)\left(-Q_{\min }\right)^{j-k}\left(\Sigma_{i n, k}^{D}\right),
$$

where $c_{j}^{\ell}$ is given by Eq. (40).

The map for the hybrid elastic DCS is given by

$$
\mu^{\prime}(\mu)=\frac{\mu^{*}-1}{2}(1-\mu)+1,
$$

where $\mu$ is on $[-1,1]$ and $\mu^{\prime}$ is on $\left[\mu_{0}^{*}, 1\right]$. Given this mapping, the moments are related by

$$
\hat{\Sigma}_{e l, \ell}=\sum_{n=0}^{\ell} c_{n}^{\ell} \frac{(-1)^{n}}{n !}\left(\frac{2}{1-\mu^{*}}\right)^{n} \sum_{k=0}^{n} b_{k}^{n} \Sigma_{e l, k}^{D}
$$

where $b_{k}^{n}$ is

$$
b_{k}^{n}=\int_{-1}^{1} P_{k}(\mu)(1-\mu)^{n} d \mu=\sum_{m=0} c_{m}^{k} \frac{(-1)^{m}}{m !} \frac{2^{n+m+1}}{n+m+1}
$$

and the coefficient $c_{m}^{k}$ or $c_{n}^{\ell}$ is given in Eq. (40).

\section{Implementation of the Moment-Preserving method classes within the Geant4 Toolkit}

Reduced order physics DCS models are central to the Moment-Preserving method and therefore, the majority of the development effort is in generating, storing, and accessing the ROP DCSs. Geant4 considers all physical interactions as processes, requiring implementation of the G4VEmProcess class, and the details of each process is captured by the model (see Apostolakis et. al. [30] the G4VEmModel class, which is the primary source of development for the MP 
method (assuming the ROP DCS is available in the form of a DCS data library). The reason that the majority of the development occurs when implementing the G4VEmModel class is because it is at this level where the ROP DCSs are stored in Geant4 data classes, and also it is at this level where the ROP DCSs are accessed.

The remaining sections provide detail on the associated physics process and model classes, the cross-section construction classes, and the cross-section library and data processing tools.

\subsection{Physics processes}

Physics processes for discrete and hybrid DCSs were implemented and are listed in Table 1 . The physics process is relatively simple because use is made of many of the virtual methods in G4VEmProcess. The only methods that required implementation include a method to initialize the process class (this consists of constructing the associated physics model and setting a few data members) and a method that determines the applicable particles.

\subsection{Physics models}

Physics models for discrete and hybrid DCSs were implemented and are listed in Table 1. Three important methods are included in the physics model class: an initialization method where the cross-section data is read in; a method for obtaining the total cross section; and a method for sampling the DCS. Use of the ROP DCS data reduces runtime and pre-existing Geant4 data tools were utilized that reduced development overhead and eased integration of the models into the toolkit. The code required to utilize the Geant4 data classes is left to section 6.3, which describes the use of the Geant4 data classes in greater detail.

There are two methods remaining for the physics model discussion, a method for obtaining the ROP total cross section and a method for sampling the ROP DCS. Obtaining the total cross section is trivial and requires a Table look-up as a function of energy and then linear interpolation on log-log scale (some minor modifications to the Geant4 data classes were required). The method 
for sampling the DCS requires two steps. First, the energy index of the data is determined using the previously discussed binary search; however, the linear interpolation is accomplished through random sampling using the weights from a linear interpolation on log-log scale [5]. Given ROP DCS evaluated at some energy grid point, the scattering angle is determined through inverting a discrete $\mathrm{CDF}$, which is a very simple process.

At this point, no mention was made of the hybrid cross section. As seen in the list of models, the hybrid cross section is composed of two models: one for soft collisions and one for hard collisions. The soft collisions are given by the discrete cross section and all of the previous discussion carries over. The hard collisions are given by the analog DCS. For the partial-wave elastic DCS much of the previous discussion also carries over. However, for the Möller inelastic DCS, an analytical expression is used to obtain the total cross section and a rejection technique is used to sample the DCS.

Table 1: List of required process and model classes for the discrete and hybrid DCSs.

\begin{tabular}{|c|c|c|}
\hline \multicolumn{3}{|c|}{ MP Class List } \\
\hline \multirow{3}{*}{ Physics Type } & Process Classes & Model Classes \\
\hline \multirow{5}{*}{ Elastic } & G4DiscreteElasticProcess & G4DiscreteElasticModel \\
& G4HybridSoftElasticProcess & G4HybridSoftElasticModel \\
& G4HybridHardElasticProcess & G4HybridHardElasticModel \\
\hline & G4DiscreteInelasticProcess & G4DiscreteInelasticModel \\
& G4HybridSoftInelasticProcess & G4HybridSoftInelasticModel \\
& G4HybridHardInelasticProcess & G4HybridHardInelasticModel \\
\hline
\end{tabular}

\subsection{Cross-section library and data processing}

An ROP cross-section library was generated for the partial-wave and Möller DCS for 1, 2, 4, and 8 points and weights. The libraries are formatted such that the Geant4 data classes could be used. For each material and number of points 
and weights, there are two data files: one for the total cross section and one for the CDF. The files are named accordingly. For example, for a 2-angle discrete DCS based on the partial-wave DCS for aluminum the two files are named gbfp_pwe_tcs_13_2.dat and gbfp_pwe_cdf_13_2.dat, where the first number is the atomic number and the second number is the number of points and weights.

There are two primary Geant 4 data classes utilized in processing and storing the ROP DCS data: G4ElementData and G4PhysicsVector. G4ElementData is a very powerful class that stores data for a particular element and then only requires the atomic number to retrieve the data. Upon construction, the G4ElementData object requires the atomic number and the data in the form of a pointer to a G4PhysicsVector object, so the G4ElementData class is really a con275 tainer of G4Physics Vector objects. The data is stored in the G4Physics Vector object, which is also a very powerful class because as long as the data is properly formatted, one must simply pass the G4PhysicsVector object a stream of the data.

Given a brief description of the implementation of the MP method within the Geant4 toolkit, we now present results. 


\section{Results}

The purpose of this paper is to demonstrate the efficacy of an implementation of the MP method within a widely used Monte Carlo particle simulation code. To do so, the MP method was first implemented in the Geant4 toolkit, as to demonstrate some of the key features of the method.

We first demonstrate that the accuracy of the method is systematically controllable through use of the MP method in estimating reflected and transmitted angular distributions and energy spectra and also to estimate longitudinal and der strict problem conditions, the accuracy of the models can be refined such that agreement with the analog benchmark is achieved. In addition, we show that for thicker slabs, or longer pathlengths, lower-order ROP DCS models are sufficiently accurate and very efficient. heterogeneous media (for one-dimensional dose deposition results see Franke and Prinja [12]). We show that accurate and efficient ROP DCS models are available for estimating dose in multiple dimensions. Moreover, we highlight another desirable feature of the MP method. That is, the ROP DCS models can dependent ROP DCS models can be applied as necessary to optimize efficiency and accuracy. Furthermore, we show that when estimating dose resulting from distributed sources, low-order ROP DCS models are sufficient. Ultimately, these results indicate that an adaptive cross section selection algorithm could improve the efficacy of an already viable method.

Lastly, we show that the MP method is effective when considering secondary electron production. That is, we present both one-dimensional charge and dose deposition results for source particle energies where secondary electron production significantly impacts the charge and dose deposition. In these settings, the 


\subsection{Thin slab results}

We first demonstrate that the accuracy of the method is systematically controllable through use of the MP method in estimating reflected and transmitted angular distributions and energy spectra in slabs with varying thicknesses. The purpose of this exercise is to show that under strict problem conditions (that is, highly peaked scattering in thin slabs) the ROP DCSs can be refined such that analog level accuracy is maintained, while still achieving efficiency gains over the analog Monte Carlo simulation. Moreover, it shown that in thicker slabs where electrons suffer further collisions, nearly-singular and highly-peaked boundary conditions are relaxed. So, use of lower-order ROP DCSs is effective. Furthermore, the presence of discrete artifacts are unavoidable when using low-order discrete DCSs to estimate angular distributions, energy-spectra, and longitudinal and lateral distributions under strict problem conditions. That said, the hybrid model can used as a means to mitigate the discrete artifacts when they are present.

\subsubsection{Reflected and transmitted angular distributions}

Reflected and transmitted angular distributions are presented below for onedimensional slabs composed of aluminum or gold with thicknesses of 100 to 3000 mfps (that is, $L=100 \lambda_{e l}, 1000 \lambda_{e l}$, and so on). The source is positioned at $x=0$ with a direction of $\vec{\Omega}=(1,0,0)$. A total of $4 \times 10^{7}$ source particles are simulated when calculating the angular distributions. Uncertainties associated with these results are within $1 \%$ in most bins, such that one can state conclusively that good agreement exists between the ROP models and the analog benchmark.

We begin by presenting transmitted angular distributions for $10-\mathrm{MeV}$ electrons on an aluminum slab $2.14 \times 10^{-2} \mathrm{~mm}(100 \mathrm{mfps})$ thick and $1-\mathrm{MeV}$ electrons on a gold slab $5.9 \times 10^{-2} \mathrm{~mm}(3000 \mathrm{mfps})$ thick in Figs. 2a and $2 \mathrm{~b}$. The result in Fig. 2a indicates that it is possible to resolve angular distributions in highly-peaked scattering regimes by systematically increasing the accuracy of the ROP DCS through preservation of additional moments. Clearly, the discrete ROP DCS model results in artifacts. The discrete aritifacts, in turn, are 
a result of both the extreme forward peakedness and the discrete form of the DCS. Under these conditions, electrons tend to suffer only a few discrete collisions before transmitting, and are scattered through preferential discrete angles corresponding to the peaks or artifacts in the angular distribution in Fig. 2a. However, use of a hybrid ROP DCS mitigates the discrete artifacts. Efficiency gains of roughly a factor of two times faster than analog Monte Carlo were realized. The result in Fig. $2 \mathrm{~b}$ provides a sense of the accuracies associated with a less-extreme scattering regime for $1-\mathrm{MeV}$ electrons on gold. Here the discrete artifacts resulting from a single-angle, single-energy discrete model are almost negligible and the general behavior of the transmitted angular distribution is captured, while remaining nearly 500 times more efficient than the analog simulation. By adding an additional angle, agreement with the analog benchmark is greatly improved, and this model is still roughly 250 times faster than the analog simulation.

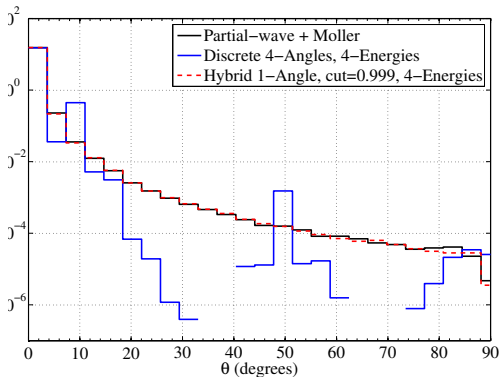

(a) $10-\mathrm{MeV}$, aluminum, $100 \mathrm{mfp}$

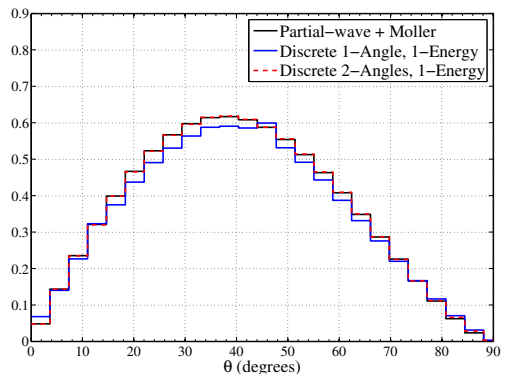

(b) 1-MeV, gold, $3000 \mathrm{mfp}$

Figure 2: Comparison of transmitted angular distributions for $10-\mathrm{MeV}$ electrons on a $2.14 \times 10^{-2} \mathrm{~mm}(100 \mathrm{mfp})$ thick aluminum slab (a) and for $1-\mathrm{MeV}$ electrons on a $2.14 \times 10^{-2} \mathrm{~mm}(3000 \mathrm{mfp})$ thick gold slab (b) calculated using the analog model (solid black) and various discrete and hybrid models.

We now present reflected angular distributions. In Figs. 3a and 3b, reflected angular distributions for $10-\mathrm{MeV}$ electrons on an aluminum slab $2.14 \times 10^{-1} \mathrm{~mm}$ (1000 mfps) thick and 1-MeV electrons on a gold slab $5.9 \times 10^{-2} \mathrm{~mm}(3000 \mathrm{mfps})$ 
thick are presented. In Fig. 3a, we again see a significant difference when using the discrete model. As noted above, this is likely discrete artifact resulting from an infrequent, but preferential backward scattering angle. Slab thickness has a similar impact on reflected distributions as for transmitted distributions. That is, with increasing slab thickness electrons suffer more collisions before being reflected, spreading the distributions in angle. In general, the discrete model tends to have the correct behavior; however, the distribution is roughly two times greater in magnitude in the case of $10-\mathrm{MeV}$ electrons in Fig. 3a. Once again, the hybrid model can be used to improve agreement. For 1-MeV electrons on a gold slab 3000 mfps thick, both the single-angle and two-angle discrete models provide noteworthy agreement considering the aforementioned efficiency gains.

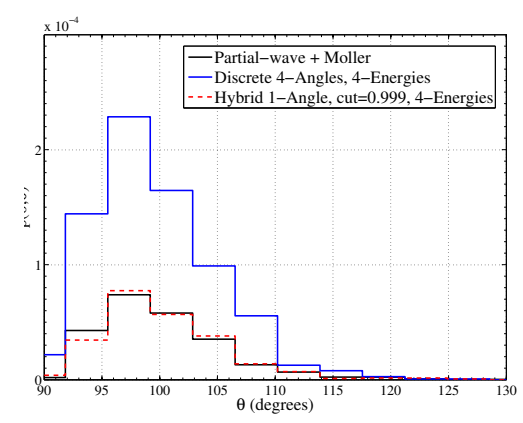

(a) 10-MeV, aluminum, $1000 \mathrm{mfp}$

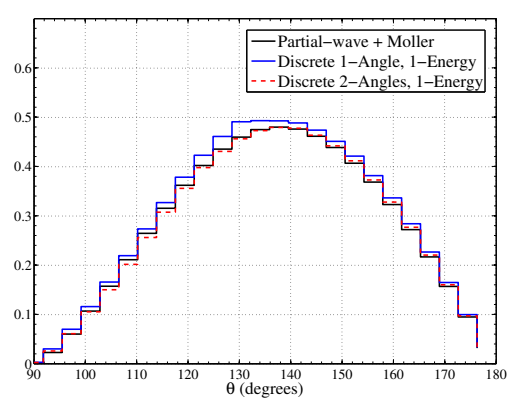

(b) 1-MeV, gold, $3000 \mathrm{mfp}$

Figure 3: Comparison of reflected angular distributions for $10-\mathrm{MeV}$ electrons on a $2.14 \times 10^{-1} \mathrm{~mm}(1000 \mathrm{mfps})$ thick aluminum slab (a) and for $1-\mathrm{MeV}$ electrons on a $5.9 \times 10^{-2} \mathrm{~mm}$ (3000 mfps) thick gold slab (b) calculated using the analog model (solid black) and various discrete and hybrid models.

\subsubsection{Energy-loss spectra}

Next, reflected and transmitted energy-loss spectra are examined. The simulation characteristics, including slab thickness, material types, and the number of source particles, are the same as described in the previous section. Dis- 
crete inelastic DCS models are not presented because a sufficient number of

ROP DCSs utilized for the $10-\mathrm{MeV}$ and the $1-\mathrm{MeV}$ problem were 12 and 90 times more efficient than the analog simulation respectively.

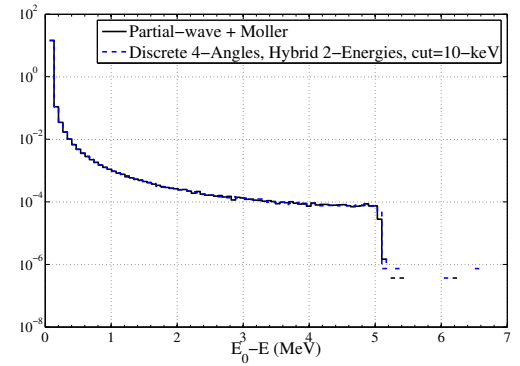

(a) $10-\mathrm{MeV}$, aluminum, $100 \mathrm{mfp}$

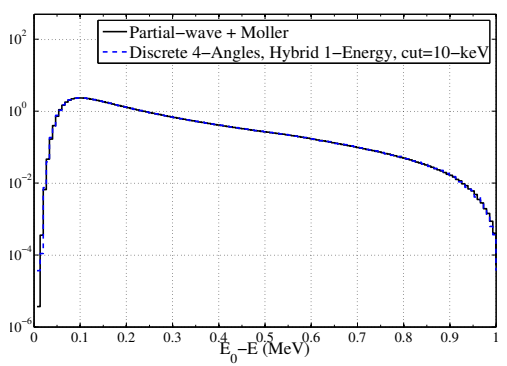

(b) $1-\mathrm{MeV}$, gold, $3000 \mathrm{mfp}$

Figure 4: Comparison of transmitted energy spectra for $10-\mathrm{MeV}$ electrons on a $2.14 \times 10^{-2} \mathrm{~mm}$ (100 mfps) thick aluminum slab (a) and for $1-\mathrm{MeV}$ electrons on a $5.9 \times 10^{-2} \mathrm{~mm}$ (3000 mfps) thick gold slab (b) calculated using the analog model (solid black) and the hybrid model.

In Figs. 5a and 5b, reflected energy-loss spectra are presented for $10-\mathrm{MeV}$ electrons on an aluminum slab $6.42 \times 10^{-1} \mathrm{~mm}(3000 \mathrm{mfps})$ thick and for $1-\mathrm{MeV}$ electrons on a gold slab $5.9 e-2 \times 10^{-2} \mathrm{~mm}(3000 \mathrm{mfps})$. In the case of $10-\mathrm{MeV}$ 
electrons, very few electrons are reflected and the tail of the energy-loss spectra are noisy. However, good agreement in the peak of the energy-spectrum can be seen. For 1-MeV electrons, significant energy-loss occurs before electrons are reflected and the energy-loss spectrum is smoother. In this case, a single-energy hybrid ROP DCS model is sufficient. Regarding efficiency gains, the problem characteristics for the $1-\mathrm{MeV}$ reflected result did not change from above and remain 90 times more efficient than the analog simulation. However, the slab thickness for the $10-\mathrm{MeV}$ reflected result changed to $3000 \mathrm{mfps}$, and efficiency gains associated with the ROP DCS models improved to roughly 250 times more efficient than the analog simulation.

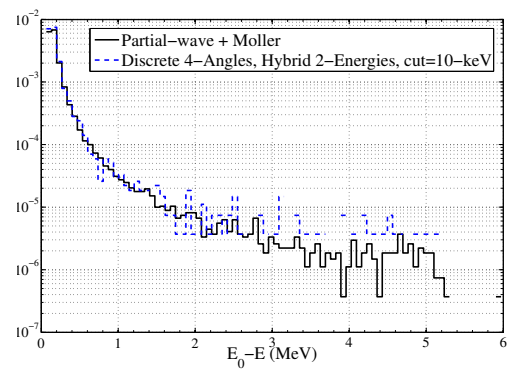

(a) $10-\mathrm{MeV}$, aluminum, $3000 \mathrm{mfp}$

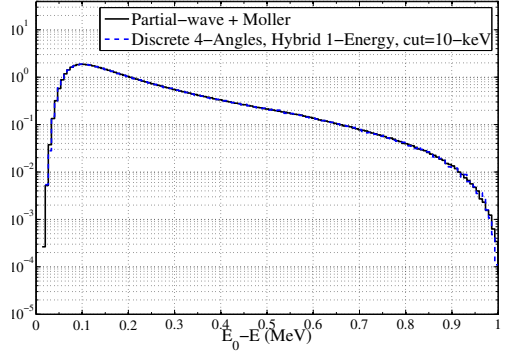

(b) 1-MeV, gold, $3000 \mathrm{mfp}$

Figure 5: Comparison of transmitted energy spectra for $10-\mathrm{MeV}$ electrons on a $6.42 \times 10^{-1} \mathrm{~mm}(3000 \mathrm{mfps})$ thick aluminum slab (a) and for $1-\mathrm{MeV}$ electrons on a $5.9 \times 10^{-1} \mathrm{~mm}(3000 \mathrm{mfp})$ thick gold slab (b) calculated using the analog model (solid black) and the hybrid model.

\subsection{Longitudinal and lateral distributions}

At this point, it is clear that accuracy and efficiency is problem dependent. Nonetheless, it is possible to refine the ROP DCS such that sufficient levels of accuracy and efficiency are realized. In this section, a few additional results are presented that overlap with the previous section in the sense that the number of collisions suffered by the electron drives the efficacy and the required accuracy of the various ROP DCS models. Here, results in connection with Lewis theory are 
presented to demonstrate the moment-preserving property of this method, while again demonstrating the accuracy of this method is systematically controllable. In particular, longitudinal and lateral distributions for $1-\mathrm{MeV}$ electrons after traveling a distance of $5.6 \times 10^{-3} \mathrm{~mm}(100 \mathrm{mfps})$ and $1.72 \times 10^{-1} \mathrm{~mm}(3000$ mfps) in an infinite medium of copper are presented (similar to Benedito et al. [5]). In these problems, energy-loss is not considered. In all of the results presented in this section, $4 \times 10^{7}$ electrons were simulated for each model.

For these problems, the electron starts at $s=0$ with an initial direction and travels until reaching a distance of $s=s_{\max }$. At this point, the electrons longitudinal displacement, or the projection of the path traveled onto the initial trajectory, and lateral displacement, or the orthogonal projection of the pathlength, is tallied.

In addition to the longitudinal and lateral distributions, a few Lewis moments are compared including $\langle z\rangle$ and $\left\langle x^{2}+y^{2}\right\rangle$ or

$$
\langle z\rangle=\int_{4 \pi} d \Omega \int_{-\infty}^{\infty} d z z \psi(z, s, \vec{\Omega})
$$

and

$$
\left\langle x^{2}+y^{2}\right\rangle=\int_{4 \pi} d \Omega \int_{-\infty}^{\infty} d x \int_{-\infty}^{\infty} d y\left(x^{2}+y^{2}\right) \psi(x, y, s, \vec{\Omega}) .
$$

As predicted by Lewis theory and seen in Tables 2 and 3, models preserving at least $\Sigma_{e l, 1}$ and $\Sigma_{e l, 2}$ will preserve $\langle z\rangle$ and $\left\langle x^{2}+y^{2}\right\rangle$. Therefore, even the very efficient single-angle model will have the correct average longitudinal and lateral displacement.

Longitudinal and lateral distributions for $1-\mathrm{MeV}$ electrons in copper are 425 presented in Figs. 6 and 7. In all cases, the hybrid DCS utilized is in excellent agreement with the analog benchmark and a cut-off of 0.9 was sufficient for pathlengths of $5.6 \times 10^{-3} \mathrm{~mm}$ and $1.7 \times 10^{-2} \mathrm{~mm}$ (100 and 300 analog mfps), while a cut-off of 0.5 was sufficient for pathengths of $5.6 \times 10^{-2} \mathrm{~mm}$ and $1.7 \times$ $10^{-1} \mathrm{~mm}$ (1000 and 3000 analog $\mathrm{mfps}$ ). Moreover, discrete artifacts are only 430 present in longitudinal and lateral distributions for pathlengths of $5.6 \times 10^{-3} \mathrm{~mm}$ (100 analog mfps). In fact, a 4-angle discrete model is sufficient for resolving 
Table 2: Average longitudinal displacement, $\langle z\rangle$, for $1-\mathrm{MeV}$ electrons in copper after traveling a distance of $5.6 \times 10^{-3} \mathrm{~mm}, 1.7 \times 10^{-2} \mathrm{~mm}, 5.6 \times 10^{-2} \mathrm{~mm}$, and $1.7 \times 10^{-1} \mathrm{~mm}(100,300,1000$, and $3000 \mathrm{mfps})$.

\begin{tabular}{ccccc}
\hline & & \multicolumn{2}{c}{ Physics Model } & \\
\cline { 3 - 4 } $\begin{array}{c}\text { Particle Energy } \\
(\mathrm{keV})\end{array}$ & $\begin{array}{c}\text { Pathlength } \\
(\mathrm{mfps})\end{array}$ & Analog & 1-Angle & Rel. Unc. \\
\hline & 100 & 0.98666 & 0.98667 & 0.00001 \\
\multirow{2}{*}{1000} & 300 & 0.96069 & 0.96070 & 0.00004 \\
& 1000 & 0.87672 & 0.87680 & 0.0001 \\
& 3000 & 0.6861 & 0.6863 & 0.0007 \\
\hline
\end{tabular}

Table 3: Average lateral displacement, $\left\langle x^{2}+y^{2}\right\rangle$, for 1-MeV electrons in copper after traveling a distance of $5.6 \times 10^{-3} \mathrm{~mm}, 1.7 \times 10^{-2} \mathrm{~mm}, 5.6 \times 10^{-2} \mathrm{~mm}$, and $1.7 \times 10^{-1} \mathrm{~mm}(100,300,1000$, and $3000 \mathrm{mfps})$.

\begin{tabular}{ccccc}
\hline & & \multicolumn{2}{c}{ Physics Model } & \\
\cline { 3 - 4 } $\begin{array}{c}\text { Particle Energy } \\
(\mathrm{keV})\end{array}$ & $\begin{array}{c}\text { Pathlength } \\
\text { (mfps) }\end{array}$ & Analog & 1-Angle & Rel. Unc. \\
\hline \multirow{2}{*}{1000} & 100 & 0.154457 & 0.154456 & 0.0003 \\
& 300 & 0.016420 & 0.016415 & 0.0008 \\
& 1000 & 0.000455 & 0.000454 & 0.003 \\
& 3000 & 0.000455 & 0.000454 & 0.003 \\
\hline
\end{tabular}

longitudinal and lateral distributions for pathlengths of $5.6 \times 10^{-2} \mathrm{~mm}$ and $1.7 \times 10^{-1} \mathrm{~mm}$ (1000 and 3000 analog $\left.\mathrm{mfps}\right)$.

\subsection{2-D dose calculations}

In this section, 2-D dose results are presented. The dose results were generated using the PWE elastic scattering DCS and the Möller inelastic scattering model for the analog benchmark and the ROP DCSs were in turn, constructed from these DCSs. Secondary electron production was not considered in this 


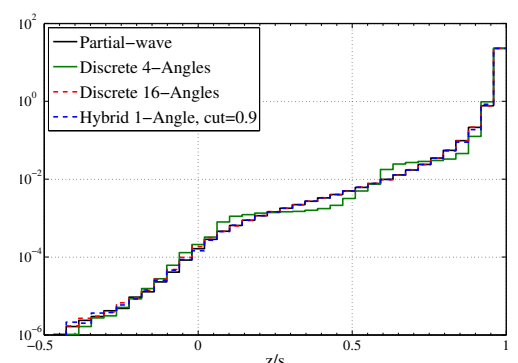

(a) $100 \mathrm{mfp}$

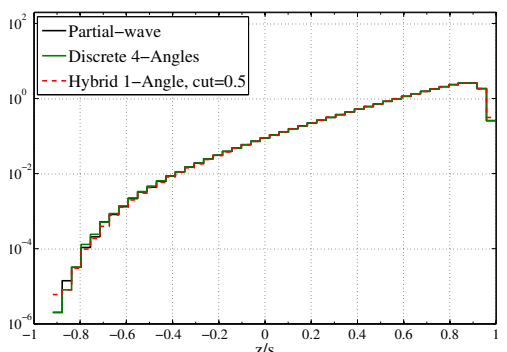

(b) $3000 \mathrm{mfp}$

Figure 6: Comparison of longitudinal distributions for $1-\mathrm{MeV}$ electrons after traveling distances of $5.6 \times 10^{-3} \mathrm{~mm}$ and $1.7 \times 10^{-1} \mathrm{~mm}$ (100 and 3000 analog elastic mfps) in copper.

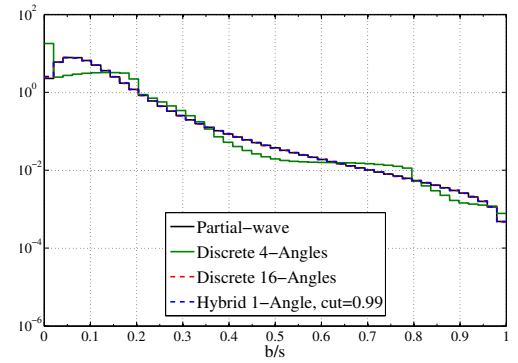

(a) $100 \mathrm{mfp}$

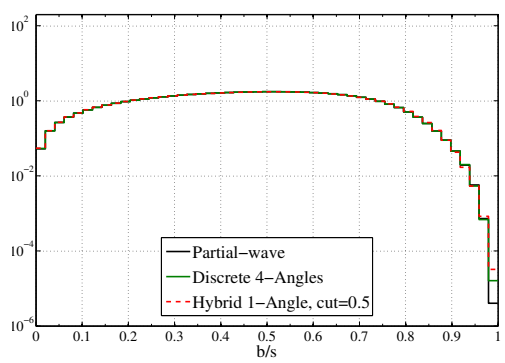

(b) $3000 \mathrm{mfp}$

Figure 7: Comparison of lateral distributions for 1-MeV electrons after traveling distances of $5.6 \times 10^{-3} \mathrm{~mm}$ and $1.7 \times 10^{-2} \mathrm{~mm}$ (100 and 3000 analog elastic mfps) in copper.

section. Two-dimensional dose deposition results are presented that include the impact of radially spreading. In particular, we show that in two-dimensions sufficiently low order discrete ROP DCS models result in discrete artifacts when transporting energetic electrons. While this could be viewed as a limitation of the method, we demonstrate a straight-forward approach to mitigating the discrete artifacts and simultaneously optimizing accuracy and efficiency. In addition, we show that accuracy of the ROP DCS models depends on the source 
configuration. That is, transporting a pencil beam of mono-energetic electrons requires higher-order models; whereas, transporting an isotropic source of electrons permits more efficient, lower-order models.

In Fig. 8a, the two-dimensional problem setup is given along with the analog benchmark in Fig. 8b. In this simulation, a beam of $10-\mathrm{MeV}$ electrons with radius of $0.02 \mathrm{~cm}$ is normally incident on a water cube with a small bone region. The analog benchmark is in logscale and provides a sense of where most of the dose is deposited. That is, a significant portion of the dose is deposited along the beamline within fractions of a $\mathrm{cm}$ to the left and right of the origin. The electrons with these energies penetrate deeply into the medium as seen in Fig. 8b.

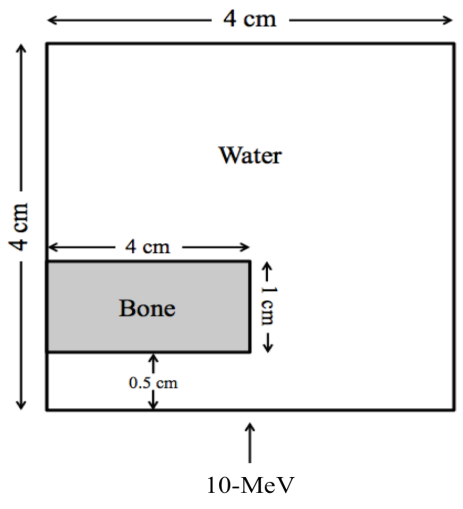

(a) 4-Angles, 4-Energies

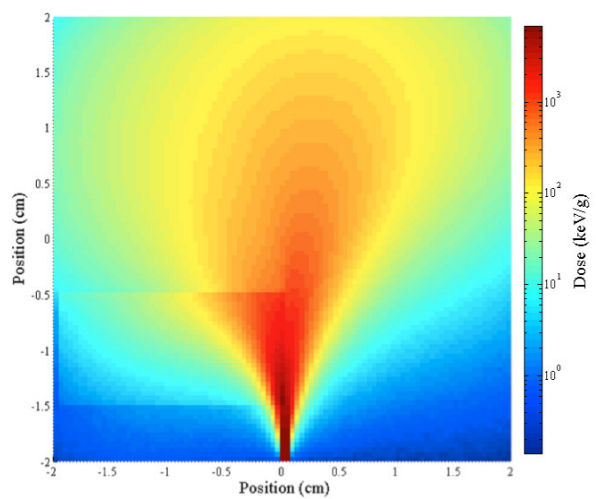

(b) 8-Angles, 4-Energies

Figure 8: The problem setup (a) and analog benchmark (b) for 10-MeV electrons in a $4-\mathrm{cm}$ by $4-\mathrm{cm}$ Water/Bone cube. The benchmark was generated using the partial-wave elastic DCS and the Möller inelastic DCS models.

The following figure presents relative error results corresponding to discrete ROP DCS models. In Fig. 9a, the relative error in the four-angle, four-energy discrete DCS models with respect to the analog benchmark is presented. Discrete artifacts can be seen clearly in Fig. 9a. However, by refining the model through preservation of additional moments with the addition of four more discrete angles, discrete artifacts are mitigated and backscatter is captured more 
accurately as seen in the relative error result in Fig. 9b. In both the four-angle and eight-angle results, no interface effects are observable. It should be noted that in the backscatter is not significant at $10-\mathrm{MeV}$ and some of the error in the lower left and right corners is statistical in nature.

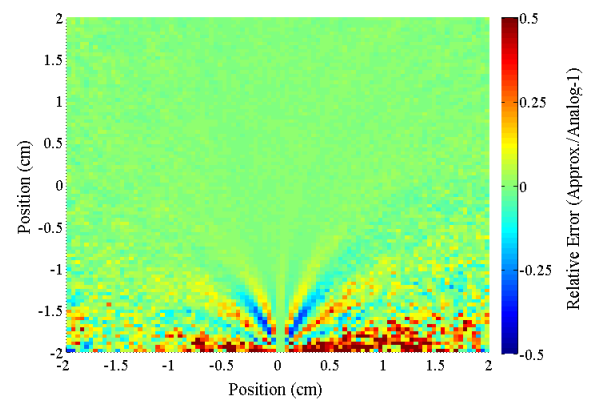

(a) 4-Angles, 4-Energies

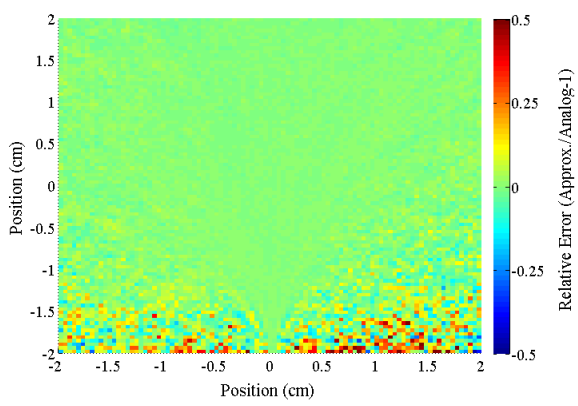

(b) 8-Angles, 4-Energies

Figure 9: The relative error in dose from $10-\mathrm{MeV}$ electrons in a $4-\mathrm{cm}$ by $4-\mathrm{cm}$ Water/Bone cube calculated using a (a) four-angle discrete elastic DCS model and four-energy discrete inelastic DCS model and a (b) eight-angle discrete elastic DCS model and four-energy discrete inelastic DCS model. 
In Fig. 10, the relative error between the analog benchmark and two hybrid models are presented. In Fig. 10a, a hybrid model with $\mu^{*}=0.9$ shows very subtle discrete artifacts, but otherwise is in good agreement. In Fig. 10b, a hybrid model with $\mu^{*}=0.99$ does not suffer from any discrete artifacts and the only disagreement is in the lower left and right corners where again the error is statistical in nature.

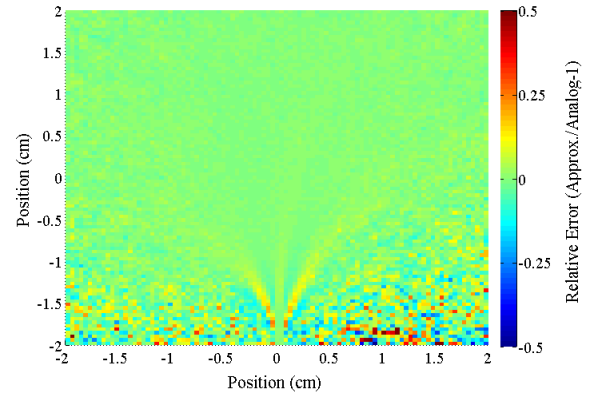

(a) Hybrid $\mu^{*}=0.9$, 4-Energies

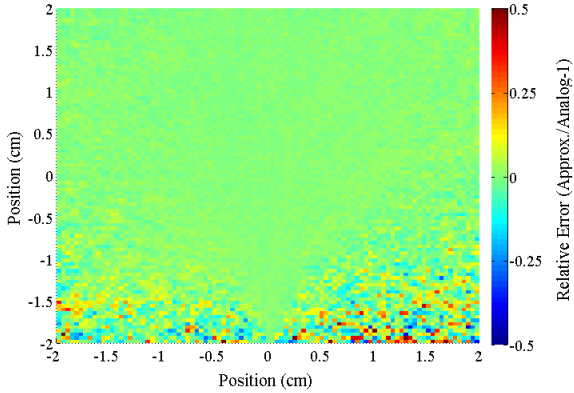

(b) Hybrid $\mu^{*}=0.99$, 4-Energies

Figure 10: The relative error in dose from $10-\mathrm{MeV}$ electrons in a $4-\mathrm{cm}$ by 4 cm Water/Bone cube calculated using a (a) single-angle hybrid elastic DCS model with $\mu^{*}=0.9$ and four-energy discrete inelastic DCS model and a (b) single-angle hybrid elastic DCS model with $\mu^{*}=0.99$ elastic DCS model and four-energy discrete inelastic DCS model.

In adding more discrete angles, or through use of the hybrid model, we demonstrated an approach to mitigate discrete artifacts through controlling the accuracy of the ROP DCS models. The only drawback to applying a more accurate ROP DCS model is the loss of efficiency as presented in Table 4. Here, the 4 -angle model is the most efficient as expected. Inclusion of additional discrete angles or use of the hybrid model reduces the efficiency gain from roughly 120 to between 47 and 94 .

As previously noted, it is possible to optimize such that significant reduction in efficiency is not incurred by applying higher-order models in regions nearby the source where the solution remains highly peaked and applying lower-order 
Table 4: Speed-up factors for various discrete DCSs when calculating dose from $10-\mathrm{MeV}$ electrons incident on a $4-\mathrm{cm}$ by $4-\mathrm{cm}$ water cube with small bone region.

\begin{tabular}{|c|c|c|c|c|}
\hline \multirow[b]{2}{*}{ Particle Energy } & \multicolumn{4}{|c|}{ ROP Model } \\
\hline & $\begin{array}{l}\text { 4-Angles } \\
\text { 4-Energies }\end{array}$ & $\begin{array}{l}\text { 8-Angles } \\
\text { 4-Energies }\end{array}$ & $\begin{array}{c}1 \text {-Angle } \\
\mu^{*}=0.9 \\
4 \text {-Energies }\end{array}$ & $\begin{array}{c}\text { 1-Angle } \\
\mu^{*}=0.99 \\
\text { 4-Energies }\end{array}$ \\
\hline $10-\mathrm{MeV}$ & 120.7 & 85.6 & 93.9 & 47.0 \\
\hline
\end{tabular}

models in regions where the solution is less-peaked. The following results present region dependent elastic ROP DCSs.

In Fig. 11, the schematic for a region dependent discrete elastic model is presented along with the associated relative error from such an approach. As seen in Fig. 11a, default 4-angle, 4-energy discrete model is applied to all regions. The default is then deactivated in the region bounded by the red dashed line and an 8-angle, 4-energy discrete model is applied in this region. The associated relative error is given in Fig. 11b. The relative error in Fig. 9b is nearly indistinguishable from the relative error in Fig. 9b where an 8-angle discrete model is applied everywhere. The resulting gain in efficiency is 106 times faster than the analog simulation as opposed to 86 when applying an 8-angle model to all regions.

In Fig. 12, the schematic for another region-dependent discrete elastic model is presented along with the associated relative error from such an approach. Again, in this problem a 4-energy discrete inelastic model is used in all regions. As seen in Fig. 12a, an 8-angle model is applied in the region where the peak dose occurs and a single-angle model is applied in all other regions. The associated relative error is given in Fig. 12b and is a modest improvement over the relative error in Fig. 11b as the backscatter is captured more accurately. Again, the relative error in Fig. 12b is nearly indistinguishable from the relative error in Fig. 9b where an 8-angle discrete model is applied everywhere. The resulting gain in efficiency is 97 times faster than the analog simulation and is reduced 


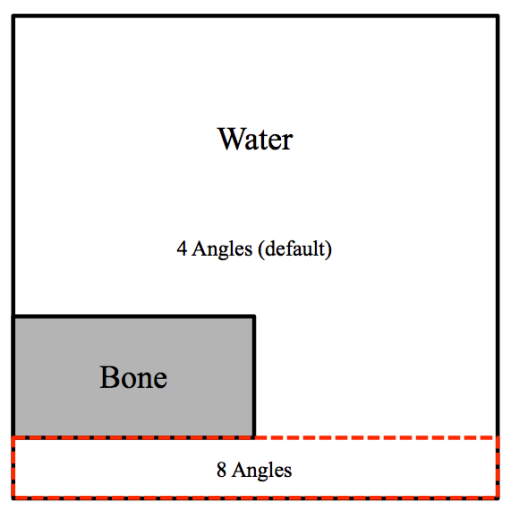

(a) Region dependent ROP DCSs

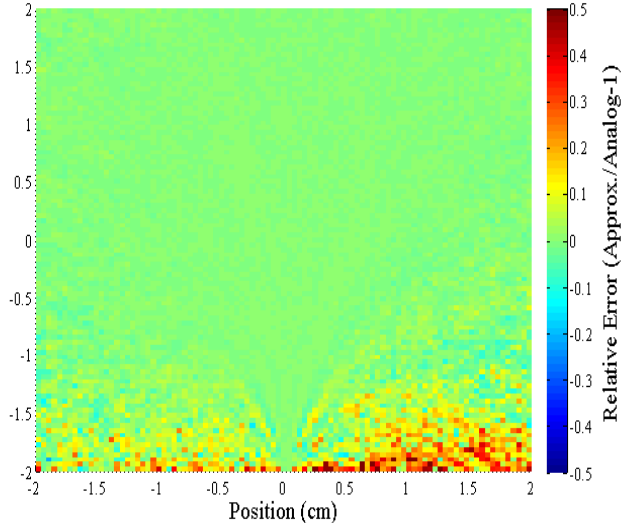

(b) Relative error

Figure 11: The schematic of region dependent ROP DCS (a) and the relative error (b) in dose from $10-\mathrm{MeV}$ electrons in a $4-\mathrm{cm}$ by 4- $\mathrm{cm}$ Water/Bone cube calculated using the region dependent ROP DCS 8-angles applied near the source and 4-angles applied in all other regions. A 4-energy discrete inelastic model is used throughout.

slightly from 106, which was efficiency gain associated with the previous region dependent models.

Ultimately, a region dependent application of the ROP DCS models is simply an exercise in demonstrating that accuracy and efficiency can be optimized. In practice, a more suitable approach would be to develop an algorithm that determines the optimal DCS model as the electron is transported. At this point, it is unclear what metric is ideal for determining the optimal ROP DCS model because the parameter space is large. The following problem illustrates this point.

For problems with singular boundary conditions like the previous problem, 515 it is clear that high-order models are necessary nearby the source and low-order models can be used away from the source as the solution becomes less peaked. However, the same is not necessarily true for distributed sources. For example, in the following problem an isotropic point source of $2.5-\mathrm{MeV}$ electrons in a gold cube $1 \mathrm{~cm}$ on each face is simulated. The problem setup and analog benchmark 


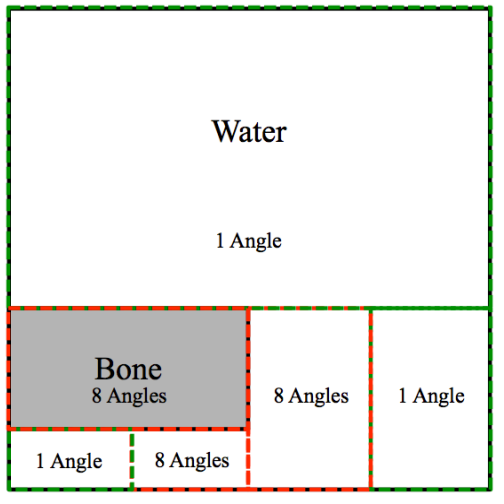

(a) Region dependent ROP DCSs

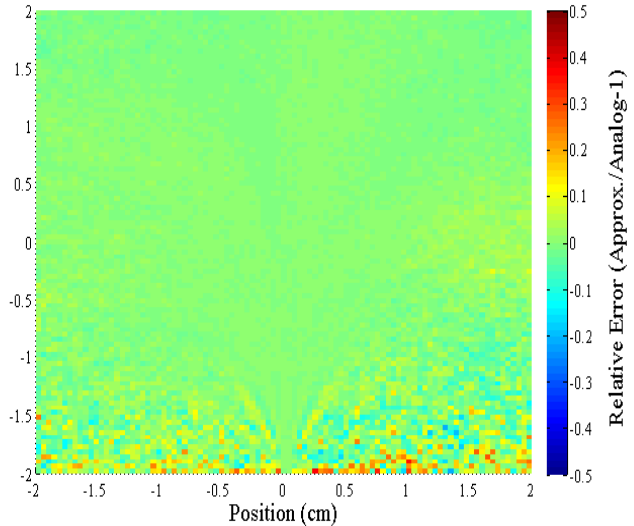

(b) Relative error

Figure 12: The schematic of region dependent ROP DCS (a) and the relative error (b) in dose from $10-\mathrm{MeV}$ electrons in a $4-\mathrm{cm}$ by $4-\mathrm{cm}$ Water/Bone cube calculated using the region dependent ROP DCS 8-angles applied in the peak dose region and 1-angle applied in all other regions. A 4-energy discrete inelastic model is used throughout.

is given in Fig. 13. As seen in Fig. 13b, the dose is deposited uniformly about the point source located at the origin. It is also of interest to point out that most of the dose is deposited nearby the source. One should also note that electron and photon transport is inherently coupled, and when simulating a photon source, the resulting source of electrons will be distributed in space, angle, and energy. Under these conditions, low-order models are likely sufficient for the transport of electrons.

As seen in Fig. 14, relatively low-order models can used to estimate the dose due to distributed sources. The most efficient model tested, single-angle, singleenergy, is about 400 times more efficient than the analog simulation and the associated relative error is presented in Fig. 14a. By adding another discrete energy the relative error improves as seen in Fig. 14b, but the efficiency decrease to about 300 times faster than the analog simulation. Finally, the most accurate model tested, 2-angles, 2-energies, is presented in Fig. 14c. This model provides good agreement, while remaining roughly 180 times more efficient than the 


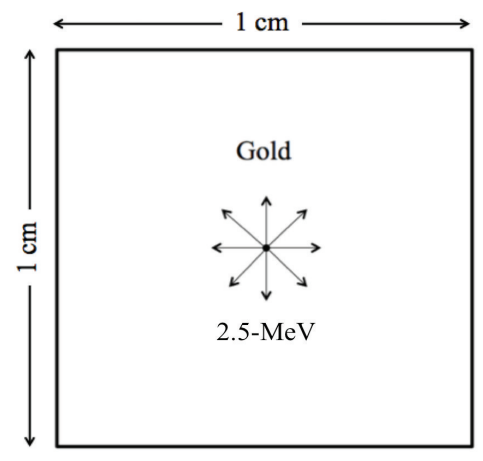

(a) Problem setup

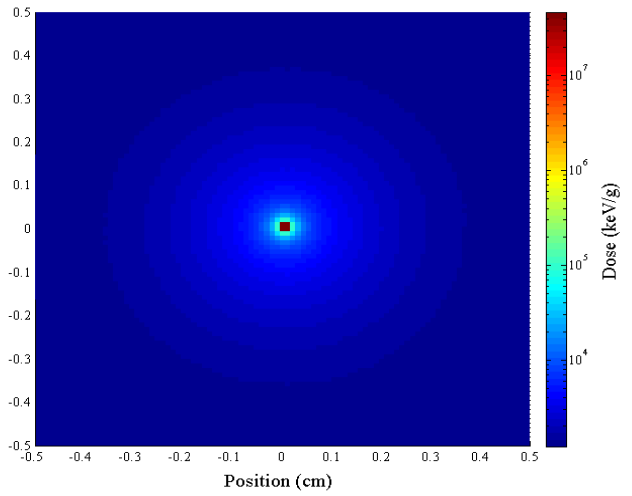

(b) Absolute dose

Figure 13: The problem setup for an isotropic point source located at the origin of a 1- $\mathrm{cm}$ by $1-\mathrm{cm}$ by 1- $\mathrm{cm}$ gold cube (a) and the analog benchmark (b) generated using the partial-wave elastic DCS and the Möller inelastic DCS models. the source configuration. Again, region dependent models could be applied in this setting for optimization, but more importantly an adaptive cross section algorithm that incorporates source information and solution information for the given problem would improve the MP method.

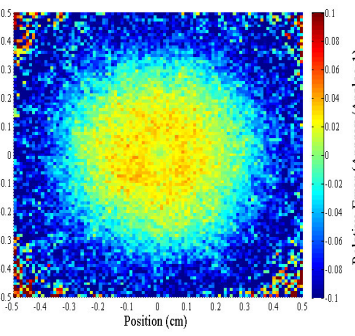

(a) 1-Angle, 1-Energy

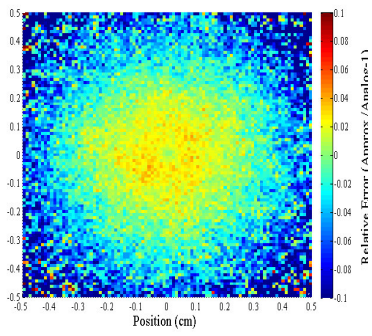

(b) 1-Angle, 2-Energies

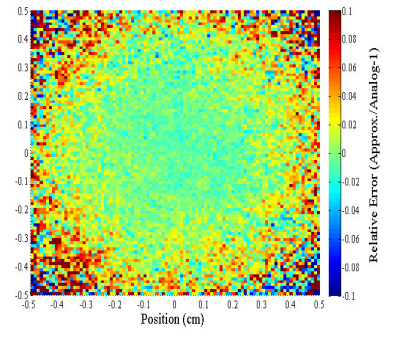

(c) 2-Angles, 2-Energies

Figure 14: The relative error in dose from $2.5-\mathrm{MeV}$ electrons in a $1-\mathrm{cm}$ by 1 $\mathrm{cm}$ by 1- $\mathrm{cm}$ gold cube calculated using (a) a single-angle, single-energy discrete model, (b) a single-angle, two-energy discrete model, and (c) a two-angle, twoenergy discrete model. 
In this section, we treat secondary electron production in the MP method through use of the discrete and hybrid ROP inelastic DCS models ${ }^{4}$. Secondary electron production occurs when the energy transferred to an atomic electron, or $Q$, is greater than the secondary electron production threshold. In the event of a secondary electron production the deflection of the primary electron and secondary electron is given by Eqs. (11) and (12). In the hybrid model secondary electron production is treated exactly through use of an analog DCS representation of the tail of the ROP DCS. However, the discrete model is an approximation of secondary electron production and requires at least a fourenergy model to provide acceptable agreement with the analog benchmark.

Figs. 15-18 include charge deposition profiles and dose deposition profiles for $5-\mathrm{MeV}$ electrons on semi-infinite aluminum and gold slabs. The uncertainty in the charge deposition and dose deposition profiles corresponds to the discrete model; however, the uncertainties corresponding to the analog model and 555 the hybrid model are comparable and bounded by the uncertainty in the discrete model. The uncertainty in the relative error figures corresponds to the uncertainty in the relative error in the hybrid model with respect to the analog benchmark. In Figs. 15b and 16b, the relative error in charge deposition for a discrete and hybrid inelastic DCS model are presented. In aluminum and gold, there is significant statistical fluctuation in the regions nearby the source where numerous secondary electrons are produced and transported away from where they are produced. In general, both the discrete and hybrid models are nearly statistically indistinguishable from the analog benchmark.

In Figs. 17b and 18b, the relative error in dose deposition for a discrete and hybrid inelastic DCS model are presented. Dose deposition is less sensitive to statistical fluctuation and the results show that inclusion of secondary electron production does not impact accuracy of the estimated dose when using the hybrid model. However, the relative error in the discrete model fluctuates

\footnotetext{
${ }^{4}$ The same discrete 4 -angle model is used throughout this section to treat elastic scattering.
} 


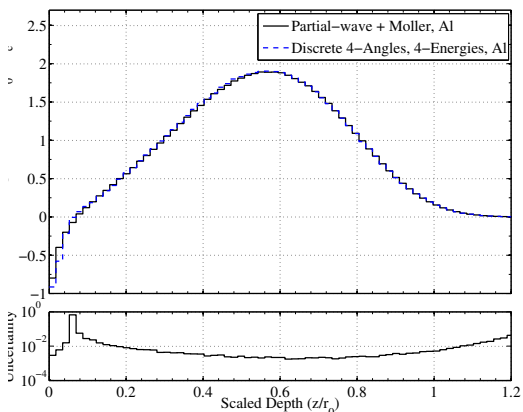

(a) Charge Deposition, Aluminum

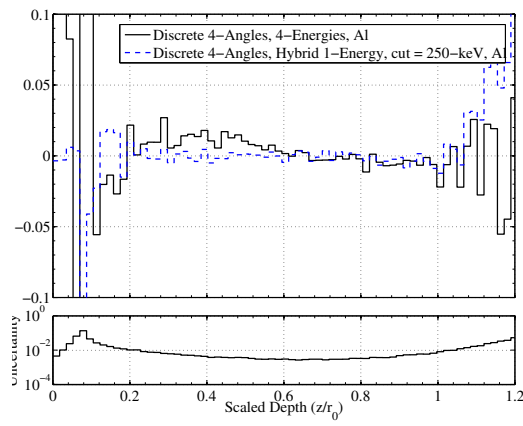

(b) Relative Error

Figure 15: Comparisons of charge deposition profiles from 5-MeV electrons on aluminum (a) and relative error (b) in discrete and hybrid models when estimating charge deposition.

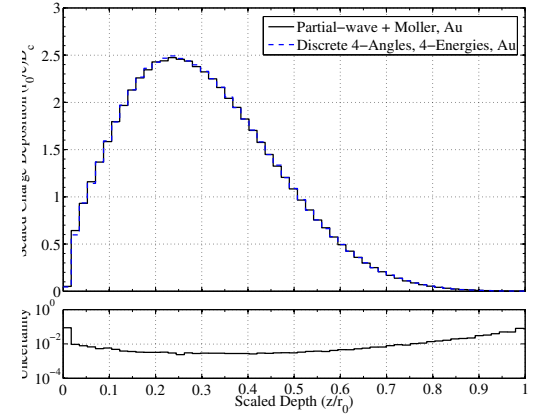

(a) Charge Deposition, Gold

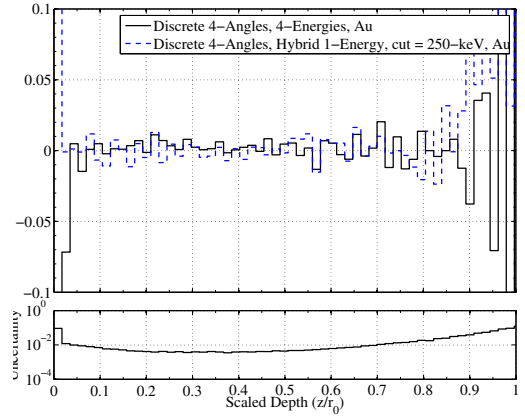

(b) Relative Error

Figure 16: Comparisons of charge deposition profiles from 5-MeV electrons on gold (a) and relative error (b) in discrete and hybrid models when estimating charge deposition.

between a few percent nearby the source, but then reduces to well within a percent throughout the remainder of the slab. Likely, the discrete model overestimates secondary electron production in the first few cells removing dose that is then deposited in the $3^{r d}$ and $4^{\text {th }}$ cell. In general, both models provide 
good agreement with the analog benchmark. However, the hybrid naturally provides improved agreement over the discrete model resulting from the form of the model.

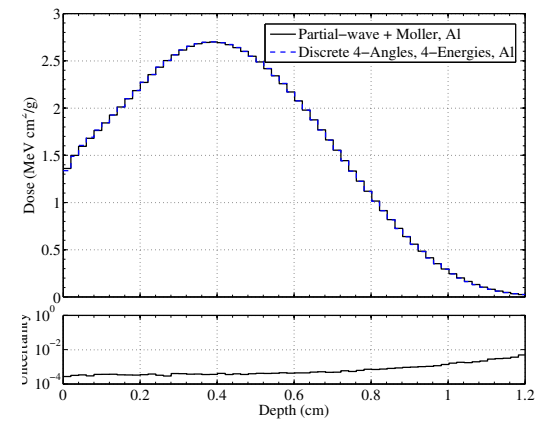

(a) Dose Deposition, Aluminum

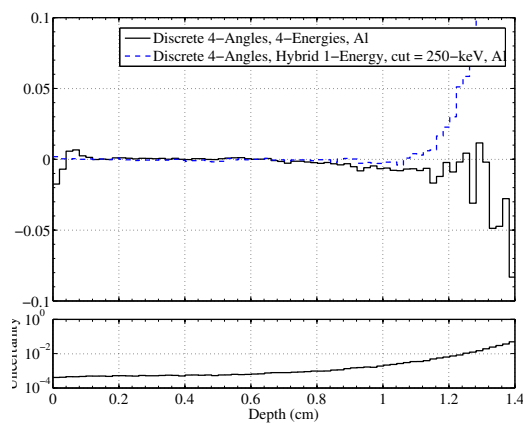

(b) Relative Error

Figure 17: Comparisons of dose deposition profiles from 5-MeV electrons on aluminum (a) and relative error (b) in discrete and hybrid models when estimating charge deposition. 


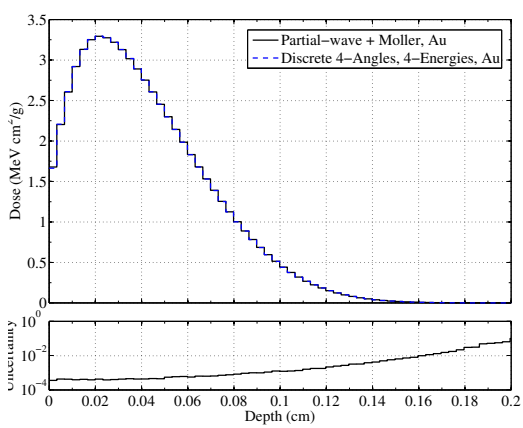

(a) Dose Deposition, Gold

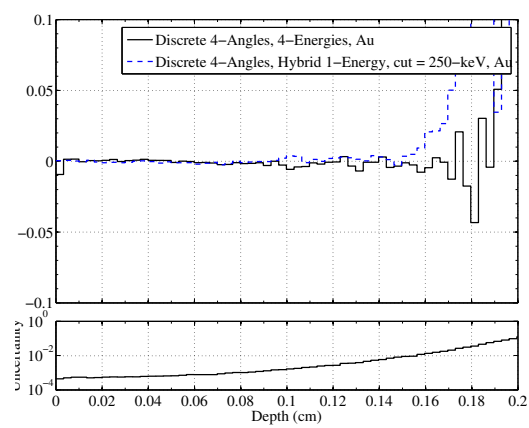

(b) Relative Error

Figure 18: Comparisons of dose deposition profiles from 5-MeV electrons on gold (a) and relative error (b) in discrete and hybrid models when estimating charge deposition. 


\section{Conclusions}

This paper serves to extend and modernize the significant body of work completed by Franke and Prinja in 2005 [12]. In extending their study we demonstrated several key features of this method including: ease of implementation in transport codes with pre-existing single scatter algorithms, independence of the method from the form of analog DCS making the method generalizable, systematically controllable accuracy while remaining efficient, and versatility through independence of the form of the analog DCS.

All of the results presented in this paper were generated using an implementation of the method within the Geant4 toolbox. Use of this method as an alternative to pre-existing scattering algorithms requires minimal implementation and maintenance effort, which is one of the unique features of this method and a result of the simple forms of the associated DCS models. When comparing the lines of code required to implement the MP method to the Geant4 $\mathrm{CH}$ method, we found that the MP method required 10 times less code.

Furthermore, we demonstrated that this method is generalizable to most single-scatter DCSs including ones in the form of tabulated DCS data like the partial-wave elastic DCSs. This is a strong feature of the method because the methods for transporting particles including total cross section look-ups or DCS sampling can be generalized when using a ROP cross-section library. That is, the algorithm is independent of the analog DCS model utilized, so improvements in the physics are captured by generating a ROP cross-section library with the most accurate scattering models available.

This method is flexible and, therefore, applicable to a wide variety of problems. By systematically controlling the accuracy the DCS models through preservation of moments, analog level accuracy on very strict test cases was achieved. In some cases efficiency gains were relatively low, but this was only true for the most stringent test where use of analog models is nearly unavoidable. We showed that in most cases, preservation of four to eight moments was sufficient for analog accuracy and efficiency gains of up to three orders of magnitude 
over analog Monte Carlo simulations were achievable. This was true when estimating a variety of quantities including angular distributions, energy spectra, longitudinal and lateral distributions, dose deposition, and charge deposition.

In the context of electron transport on modern architectures, data libraries for the PW DCSs for energy and material combinations of interest may prove to be prohibitively large. Though data reduction techniques are not studied herein, this method may provide the necessary means for data reduction required by modern architectures.

This work establishes a modern basis from which further testing of this method will be completed. In fact, a great deal of work remains in the area of experimental validation, adaptive cross section selection algorithms, and applications to protons and heavy ions. Currently, no thorough validation studies have been completed. This will require identifying and analog model that gives good agreement with various experimental benchmarks and then validation of the MP method will follow without difficulties. Furthermore, a validation study will require the demonstration of applying the MP method along with pre-existing bremsstrahlung models. Integration of the MP method with pre-existing bremsstrahlung models is assumed to be trivial, especially, if bremsstrahlung production is an exponentially distributed random process. 625 The two-dimensional dose results indicate that it is not only possible to optimize efficiency and accuracy, but that the MP method would be improved by including an adaptive algorithm for such purposes. The MP method is clearly applicable to electrons and easily extended to positrons. However, it is unclear how effective the MP method will be at representing extremely peaked scattering characteristic of protons and heavy ions. That said, it is known that the ROP DCS construction tools will successfully generate ROP DCSs for extremely peaked analog DCSs $\left(\eta \sim 10^{-16}\right)$. 


\section{Acknowledgements}

The first two authors would like to thank the AFRL for funding the grant 635 that supported this work and the UNM Center for Advanced Research Computing for providing the computational resources required to complete this work along with top-notch user support.

Sandia National Laboratories is a multi-program laboratory managed and operated by Sandia Corporation, a wholly owned subsidiary of Lockheed Martin

${ }_{640}$ Corporation, for the U.S. Departments of Energy's National Nuclear Security Administration under contract DE-AC04-94AL85000. 


\section{References}

\section{References}

[1] L. Carter, E. Cashwell, Particle transport simulation with the Monte Carlo method, Tech. Rep. TID 36607, Technical Information Division (1975).

[2] M. J. Berger, Monte Carlo calculation of the penetration and diffusion of fast charged particles, in: Methods in Computational Physics, Vol. 1 of Statistical Physics, Advances in Research and Applications, Academic Press, 1963, pp. 135-215.

[3] A. Bielajew, D. D. O. Rogers, PRESTA: The parameter reduced electronstep transport algorithm for electron Monte Carlo transport, Nucl. Instr. Meth. B 18 (1986) 165-181.

[4] I. Kawrakow, A. Bielajew, On the representation of electron multiple elastic-scattering distributions for Monte Carlo calculations, Nucl. Instr. Meth. B 134 (1998) 325-336.

[5] E. Benedito, J. M. Fernández-Varea, F. Salvat, Mixed simulation of the multiple elastic scattering of electrons and positrons using partial-wave differential cross-sections, Nucl. Instr. Meth. B 174 (2001) 91-110.

[6] M. Landesman, J. E. Morel, Angular Fokker-Planck decomposition and representation techniques, Nucl. Sci. Eng. 103 (1989) 1-11.

[7] J. E. Morel, An improved Fokker-Planck angular differencing scheme, Nucl. Sci. Eng. 89 (1985) 131-136.

[8] J. Ligou, Fusion reaction product transport in inertially confined plasmas, in: Proc. ANS-ENS Int. Topl. Mtg, Advances in Mathematical Methods for the Solution of Nuclear Engineering Problems, Fachinformationszentrum Energie,Physik, Mathematik GmbH, Karlsruhe, West Germany, 1981. 
[9] J. E. Morel, L. J. Lorence, Jr., R. P. Kensek, J. A. Hableib, D. P. Sloan, A hybrid multigroup/continuous-energy Monte Carlo method for solving the Boltzmann-Fokker-Planck equation, Nucl. Sci. Eng. 124 (1996) 369-389.

[10] A. K. Prinja, G. Pomraning, A generalized Fokker-Planck model for transport of collimated beams, Nucl. Sci. Eng. 137 (2001) 227-235.

[11] C. L. Leakas, E. W. Larsen, Generalized Fokker-Planck approximations of particle transport with highly forward-peaked scattering, Nucl. Sci. Eng. 137 (2001) 236-250.

[12] B. C. Franke, A. K. Prinja, Monte Carlo electron dose calculations using discrete scattering angles and discrete energy losses, Nucl. Sci Eng. 149 (2005) 1-22.

[13] D. Dixon, A. K. Prinja, B. C. Franke, Geant4 implementation of a novel single-event Monte Carlo method for electron dose calculations, Trans. Am. Nucl. Soc.

[14] B. C. Franke, A. K. Prinja, R. P. Kensek, L. J. Lorence, Ray effect mitigation for electron transport with discrete scattering-angles, Trans. Am. Nucl. Soc. 87 (2002) 133-135.

[15] L. Harding, Monte Carlo simulations of electron and positron energy-loss straggling, Master's thesis, University of New Mexico (2006).

[16] F. Salvat, A. Jablonski, C. J. Powell, ELSEPA - Dirac partial-wave calculation of elastic scattering of electrons and positrons by atoms, positive ions and molecules, Comp. Phys. Comm. 165 (2005) 157-190.

[17] S. Agostinelli, et al., Geant4 - a simulation toolkit, Nucl. Instr. Meth. A 506 (2003) 250-303.

[18] ICRU Report No. 77, Elastic scattering of electrons and positrons, Journal of ICRU 7. 
[19] R. Evans, The Atomic Nucleus, tenth printing Edition, McGraw-Hill, New York, 1955.

[20] ICRU Report No. 37, Stopping powers for electrons and positrons, Journal of ICRU.

[21] H. W. Lewis, Multiple scattering in an infinite medium, Phys. Rev. 78 (1950) 526-529.

[22] A. K. Prinja, Lewis theory for energy straggling in thin layers, Nucl. Instr. Meth. B 330 (2014) 99-102.

[23] B. C. Franke, A. K. Prinja, R. P. Kensek, L. J. Lorence, Discrete scatteringangle model for electron pencil beam transport, Trans. Am. Nucl. Soc. 86 (2002) 206-208.

[24] J. E. Morel, Fokker-Planck calculations using standard discrete ordinates transport codes, Nucl. Sci. Eng. 79 (1981) 340-356.

[25] D. Dixon, A computationally efficient moment-preserving monte carlo electron transport method with implementation in geant4, Ph.D. thesis, University of New Mexico (2015).

[26] W. Gautschi, Numerical Analysis, 2nd Edition, Birkhauser, New York, 2012.

[27] G. H. Golub, Some modified matrix eigenvalue problems, SIAM Rev. 15 (2) (1973) 318-334.

[28] G. H. Golub, J. H. Welsch, Questions of numerical condition related to orthogonal polynomials, Studies in Numerical Analysis 24 (1984) 140-177.

[29] G. H. Golub, J. H. Welsch, Calculation of Gauss quadrature rules, Math. Comp. 23 (106) (1969) 221-230.

[30] J. Apostolakis, et al., Geometry and physics of the geant4 toolkit for high and medium energy applications, Radiat. Phys. Chem. 78 (2009) 859-873. 
[31] V. N. Ivanchenko, et al., Recent improvements in geant4 electronmagnetic physics modeling. 